\title{
INDEPENDENCE OVER ARBITRARY SETS IN NSOP THEORIES
}

\author{
JAN DOBROWOLSKI, BYUNGHAN KIM, AND NICHOLAS RAMSEY
}

\begin{abstract}
We study Kim-independence over arbitrary sets. Assuming that forking satisfies existence, we establish Kim's lemma for Kim-dividing over arbitrary sets in an $\mathrm{NSOP}_{1}$ theory. We deduce symmetry of Kim-independence and the independence theorem for Lascar strong types.
\end{abstract}

\section{Contents}

1. Introduction

2. The existence axiom

3. Kim's lemma over arbitrary sets 9

4. Symmetry 12

5. 3-amalgamation for Lascar strong types 15

6. The skew tree property 18

References

\section{INTRODUCTION}

Kim-independence is a notion of independence that unifies and explains simplicitylike phenomena in a non-simple setting. Non-forking independence, introduced originally by Shelah, allowed for the organization of core properties of simple theories into a collection of basic principles - the 'non-forking calculus' - that made possible the deepening of simplicity theory and the streamlined treatment of examples with simple theory. Following these developments, increased attention was given to classes of theories where a notion of independence might serve a similar explanatory role. This was motivated from below by the study of independence and amalgamation in natural examples - the generic parametrized equivalence relations of Džamonja and Shelah, Granger's generic vector spaces with a bilinear form, and Frobenius fields studied by Chatzidakis - as well as from above, by the desire to situate the theorems of simplicity theory, especially those dealing with independence relations, in their broadest possible setting. It was known from the work of Chernikov and the third-named author [4] that the existence of a well-behaved

Date: September 19, 2019.

The first author was supported by European Union's Horizon 2020 research and innovation programme under the Marie Sklodowska-Curie grant agreement No 705410, by NCN Grant no. 2015/19/D/ST1/01174, and by the Foundation for Polish Science (FNP). The second author has been supported by Samsung Science Technology Foundation under Project Number SSTFBA1301-03 and an NRF of Korea grant 2018R1D1A1A02085584. 
notion of independence implies that a theory is $\mathrm{NSOP}_{1}$ and the theory of Kimindependence allows one to reverse the implication, using the hypothesis of $\mathrm{NSOP}_{1}$ alone to develop a notion of independence that specializes to natural notions of independence in the examples mentioned above. Since it was introduced in [9, it has been established that almost all of the important properties of non-forking independence in simple theories, with the exception of base monotonicity, carry over to Kim-independence in the $\mathrm{NSOP}_{1}$ setting.

However, one major limitation for the theory of Kim-independence is that the theory, up to this point, has only been developed for the special situation where one considers independence over a model. The reason for this is that Kim-independence is defined to be independence at a generic scale - it is defined in terms of Kimdividing formulas, that is formulas that divide with respect to a generic sequence. The initial definition of Kim-dividing took 'generic sequence' to mean Morley sequence in a global invariant type. In any theory, given any type over a model, one can construct a Morley sequence in a global finitely satisfiable, hence invariant, type extending it, which means that the idea of dividing along a generic sequence is not vacuous. Over arbitrary sets, it is certainly the case that one cannot expect every type to have a finitely satisfiable or even invariant global extension, but one might instead consider those theories in which every complete type has a global non-forking extension - a (non-forking) Morley sequence in this type would furnish a notion of generic sequence allowing one to define Kim-independence over arbitrary sets. This property, that every type extends to a global non-forking type, is equivalent to what we call non-forking existence, which asserts that no type forks over its domain. As far as we know, all $\mathrm{NSOP}_{1}$ theories that have been studied satisfy non-forking existence, and it is known that every $\mathrm{NSOP}_{1}$ theory is interpretable in an $\mathrm{NSOP}_{1}$ theory in which this holds [14.

Here we show that the entire theory of Kim-independence can be extended to give a notion of independence over arbitrary sets in $\mathrm{NSOP}_{1}$ theories with non-forking existence. We modify the definition of Kim-dividing so that a formula $\varphi(x ; a) \mathrm{Kim}$ divides over $A$ if it divides along some non-forking Morley sequence over $A$. In the context of an $\mathrm{NSOP}_{1}$ theory, this gives an equivalent notion of independence when $A$ is a model but this equivalence over models is obtained as a corollary after the theory of Kim-independence has been developed, using invariant types at every stage. Consequently, new methods were required in extending the theory to arbitrary sets. This is most pronounced in the proof of Kim's lemma, which reduces Kim's lemma over sets to Kim's lemma over models, instead of merely adapting the existing proof. This uses methods from classical stability, related to the fundamental order, as well as the construction of suitable trees.

As a consequence of the theory of independence developed here, we prove independent 3-amalgamation for Lascar types in $\mathrm{NSOP}_{1}$ theories with non-forking existence. In simple theories, the independence theorem over a model was proved in [13, from which 3-amalgamation for Lascar types was deduced as a corollary. Later, Shami proved 3-amalgamation for Lascar types in a simple theory directly [16], which has the result 'over models' as an immediate consequence. Shami's proof influences the approach to 3-amalgamation for $\downarrow^{K}$ taken here, but we still proceed by deducing the theorem from the independence theorem over models for $\downarrow^{K}$ in $\mathrm{NSOP}_{1}$ theories. Indeed, our proof relies on Kim's lemma, extension, and the symmetry of Kim-dividing over arbitrary sets in $\mathrm{NSOP}_{1}$ theories with existence, and the 
independence theorem over models for $\downarrow^{K}$ is an essential ingredient in the proofs of these properties. It seems unlikely that there is a direct proof of 3-amalgamation over sets without appealing to the corresponding result over models in $\mathrm{NSOP}_{1}$ theories. Lastly, we mention that any simple theory is $\mathrm{NSOP}_{1}$ with non-forking existence and in a simple theory, Kim-independence and non-forking independence coincide. Our results, therefore, generalize these theorems from simplicity theory.

In the final section, we introduce a syntactic property called the skew tree property, or STP, which is equivalent to $\mathrm{NSOP}_{1}$ among theories satisfying non-forking existence and may be characterized by a certain amalgamation property. The general question of whether STP is equivalent to $\mathrm{SOP}_{1}$ bears a resemblance to the question of whether $\mathrm{SOP}_{1}$ and $\mathrm{SOP}_{2}$ are equivalent (both are questions about whether one can 'upgrade' a tree assumed to have a limited quantity of inconsistency to one with considerably more) and offers a test case for whether a theory of independence might be useful for collapsing or separating syntactic dividing lines. We end with several open problems.

\section{The EXIsTence AXIOM}

We let $T$ be a complete theory in a language $\mathcal{L}$. We fix a monster model $\mathbb{M} \models$ $T$ which is $\bar{\kappa}$-saturated and $\bar{\kappa}$-homogeneous for some sufficiently large $\bar{\kappa}$-we will implicitly assume all models and parameter sets are of size $<\bar{\kappa}$ and contained in M.

2.1. Forking and dividing. We recall the following standard definitions:

Definition 2.1. Let $A$ be a set of parameters.

(1) Let $k$ be a natural number. We say a formula $\varphi\left(x, a_{0}\right) k$-divides over $A$ if for some $A$-indiscernible sequence $\left\langle a_{i}: i<\omega\right\rangle$ in $\operatorname{tp}\left(a_{0} / A\right),\left\{\varphi\left(x, a_{i}\right) \mid i<\omega\right\}$ is $k$-inconsistent (meaning its each $k$-element subset is inconsistent).

(2) A formula divides over $A$ if it $k$-divides over $A$ for some $k$.

(3) A formula $\varphi(x ; a)$ forks over $A$ if $\varphi(x ; a) \vdash \bigvee_{i<k} \psi_{i}\left(x ; b_{i}\right)$ where $\psi_{i}\left(x ; b_{i}\right)$ divides over $A$ for each $i<k$.

(4) A type $p(x)$ forks or divides over $A$ if it implies a formula that forks or divides over $A$, respectively.

(5) We write $a \downarrow_{A} b$ and $a \downarrow_{A}^{d} b$ to denote the assertions that $\operatorname{tp}(a / A b)$ does not fork over $A$ and does not divide over $A$, respectively .

We will make free use of the following facts about the relation $\downarrow$ (see, e.g. [11]):

Fact 2.2. Let $A$ be a set of parameters.

(1) Extension: For any $c$, if $a \downarrow_{A} b$, there is $a^{\prime} \equiv_{A b} a$ so that $a^{\prime} \downarrow_{A} b c$.

(2) Left-transitivity: If $a \downarrow_{A} c$ and $b \downarrow_{A a} c$ then $a b \downarrow_{A} c$.

(3) Base monotonicity: If $a \downarrow_{A} b c$ then $a \downarrow_{A b} c$.

(2) and (3) hold for $\downarrow^{d}$ as well.

Definition 2.3. 11, Definition 2.2.3] Let $A \subseteq B$ and $p \in S(B)$. By a Morley sequence in $p$ over $A$, we mean a $B$-indiscernible sequence $\left\langle a_{i}: i<\omega\right\rangle$ of realizations of $p$ such that for every $i<\omega, \operatorname{tp}\left(a_{i} / B a_{<i}\right)$ does not fork over $A$. When $A=B$, we omit mentioning over $A$.

In order to construct Morley sequences, we will often use the following consequence of the Erdős-Rado theorem: 
Fact 2.4. 17. Let $B$ be a set of parameters and $\kappa$ a cardinal. For any sequence $\left(a_{i}\right)_{i<\beth\left(\left(2^{|T|+|B|+\kappa}\right)^{+}\right)}$consisting of tuples of length $\leq \kappa$ there is a $B$-indiscernible sequence $\left(a_{j}^{\prime}\right)_{j<\omega}$ based on $\left(a_{i}\right)_{i<\beth\left(2^{|T|+|B|+\kappa}\right)^{+}}$over $B$, i.e., : For every $k<\omega$ there are $i_{0}<i_{1}<\cdots<i_{k-1}$ such that $a_{0}^{\prime} a_{1}^{\prime} \ldots a_{k-1}^{\prime} \equiv_{B} a_{i_{0}} a_{i_{1}} \ldots a_{i_{k-1}}$.

Fact 2.5. (1) [11, Lemma 2.2.5] Let $I=\left\langle a_{i}\right\rangle_{i}$ be a Morley sequence in $p=$ $\operatorname{tp}\left(a_{i} / A\right)$, and let $J=\left\langle b_{j}\right\rangle_{j}$ be an arbitrary $A$-indiscernible in $p$. Then there is $I^{\prime} \equiv_{A} I$ such that $b_{j} I^{\prime}$ is an $A$-indiscernible for each $b_{j}$.

(2) Let $I^{\frown} J$ be a Morley sequence over $A$. Then due to left-transitivity and base monotonicity of nonforking independence, we have $J \downarrow_{A} I$.

Recall that the existence axiom (with respect to nonforking independence) says that any complete type over a set does not fork over the set, and that a global type is a type in $S(\mathbb{M})$.

Remark 2.6. The following are equivalent.

(1) $T$ satisfies the existence axiom.

(2) For all parameter sets $A$, no consistent formula over $A$ forks over $A$.

(3) For all parameter sets $A$, every type $p \in S(A)$ has a global extension that does not fork over $A$.

(4) For all parameter sets $A$ and any $p \in S(A)$, there is a Morley sequence in $p$.

Proof. (1) $\Longrightarrow(2)$ is clear.

$(2) \Longrightarrow(3)$ : given a type $p \in S(A)$, consider the partial type

$$
p(x) \cup\{\neg \varphi(x ; c): \varphi(x ; c) \text { forks over } A\} .
$$

By compactness, if this partial type is inconsistent, there is some $\psi(x) \in p$ such that

$$
\psi(x) \vdash \bigvee_{i<k} \varphi_{i}\left(x ; c_{i}\right)
$$

where each $\varphi_{i}\left(x ; c_{i}\right)$ forks over $A$, which means that $\psi(x)$ is a formula over $A$ that forks over $A$, contradicting (2). Therefore this partial type is consistent, and any completion gives the desired global extension.

$(3) \Longrightarrow(4)$ Given $p$, let $q$ be a global extension that does not fork over $A$. Let $\kappa$ be sufficiently large and choose the sequence $\left\langle a_{i}: i<\kappa\right\rangle$ so that $\left.a_{i} \models q\right|_{A a_{<i}}$. Then $a_{i} \downarrow_{A} a_{<i}$ for all $i<\kappa$. Applying Lemma 2.4, we obtain the desired Morley sequence.

$(4) \Longrightarrow(1)$ : If $p$ is an arbitrary complete type over $A$, let $\left\langle a_{i}: i<\omega\right\rangle$ be a Morley sequence in $p$. Then in particular, $a_{1} \models p$ and $a_{1} \downarrow_{A} a_{0}$, which implies $a_{1} \downarrow_{A} A a_{0}$ and hence $a_{1} \downarrow_{A} A$. This shows that the existence axiom is satisfied.

A global type $q \in S(\mathbb{M})$ is called $A$-invariant if $b \equiv_{A} b^{\prime}$ implies $\varphi(x ; b) \in q$ if and only if $\varphi\left(x ; b^{\prime}\right) \in q$. A global type $q$ is invariant if there is some small set $A$ such that $q$ is $A$-invariant.We write $a \downarrow_{M}^{u} B$ to mean $\operatorname{tp}(a / M B)$ is finitely satisfiable in $M$, in other words $\operatorname{tp}(a / M B)$ is a coheir of its restriction to $M$. We say $\operatorname{tp}(a / M B)$ is an heir of its restriction to $M$ if $B \downarrow_{M}^{u} a$.

Definition 2.7. Suppose $q$ is an $A$-invariant global type and $I$ is a linearly ordered set. By a Morley sequence in $q$ over $A$ of order type $I$, we mean a sequence $\left\langle b_{\alpha}: \alpha \in I\right\rangle$ such that for each $\alpha \in I,\left.b_{\alpha} \models q\right|_{A b_{<\alpha}}$ where $b_{<\alpha}=\left\langle b_{\beta}: \beta<\alpha\right\rangle$. 
Given a linear order $I$, we will write $q^{\otimes I}$ for the global $A$-invariant type in variables $\left\langle x_{\alpha}: \alpha \in I\right\rangle$ such that for any $B \supseteq A$, if $\left.\bar{b} \models q^{\otimes I}\right|_{B}$ then $\left.b_{\alpha} \models q\right|_{B b_{<\alpha}}$ for all $\alpha \in I$. If $q$ is, moreover, finitely satisfiable in $A$, in which case $b_{\alpha} \downarrow_{A}^{u} b_{<\alpha}$ for all $\alpha \in I$, then we refer to a Morley sequence in $q$ over $A$ as a coheir sequence over $A$. Likewise, an $A$-indiscernible sequence $\left\langle a_{i}: i<\omega\right\rangle$ such that $a_{<i} \downarrow_{A}^{u} a_{i}$ is called an heir sequence over $A$.

\subsection{NSOP$_{1}$ and Kim-independence.}

Definition 2.8. [8, Definition 2.2] The formula $\varphi(x ; y)$ has $\mathrm{SOP}_{1}$ if there is a collection of tuples $\left(a_{\eta}\right)_{\eta \in 2}<\omega$ so that

- For all $\eta \in 2^{\omega},\left\{\varphi\left(x ; a_{\eta \mid \alpha}\right): \alpha<\omega\right\}$ is consistent.

- For all $\eta \in 2^{<\omega}$, if $\nu \unrhd \eta \frown\langle 0\rangle$, then $\left\{\varphi\left(x ; a_{\nu}\right), \varphi\left(x ; a_{\eta \frown 1}\right)\right\}$ is inconsistent. We say $T$ is $\mathrm{SOP}_{1}$ if some formula has $\mathrm{SOP}_{1}$ modulo $T$. $T$ is $\mathrm{NSOP}_{1}$ otherwise.

Fact 2.9. [4, Lemma 5.1] 9, Proposition 2.4] The following are equivalent:

(1) $T$ has $\mathrm{SOP}_{1}$

(2) There is a formula $\varphi(x ; y)$ and an array $\left(c_{i, j}\right)_{i<\omega, j<2}$ so that

(a) $c_{i, 0} \equiv_{\bar{c}_{<i}} c_{i, 1}$ for all $i<\omega$.

(b) $\left\{\varphi\left(x ; c_{i, 0}\right): i<\omega\right\}$ is consistent.

(c) $\left\{\varphi\left(x ; c_{i, 1}\right): i<\omega\right\}$ is 2 -inconsistent.

(3) There is a formula $\varphi(x ; y)$ and an array $\left(c_{i, j}\right)_{i<\omega, j<2}$ so that

(a) $c_{i, 0} \equiv_{\bar{c}_{<i}} c_{i, 1}$ for all $i<\omega$.

(b) $\left\{\varphi\left(x ; c_{i, 0}\right): i<\omega\right\}$ is consistent.

(c) $\left\{\varphi\left(x ; c_{i, 1}\right): i<\omega\right\}$ is $k$-inconsistent for some $k$.

Definition 2.10. Suppose $A$ is a set of parameters.

(1) We say a formula $\varphi\left(x, a_{0}\right)$ Kim-divides over $A$ if for some Morley sequence $\left\langle a_{i}: i<\omega\right\rangle$ in $\operatorname{tp}\left(a_{0} / A\right),\left\{\varphi\left(x, a_{i}\right) \mid i<\omega\right\}$ is inconsistent.

(2) A formula $\varphi(x ; a)$ Kim-forks over $A$ if $\varphi(x ; a) \vdash \bigvee_{i<k} \psi_{i}\left(x ; b_{i}\right)$ where $\psi_{i}\left(x ; b_{i}\right)$ Kim-divides over $A$ for all $i<k$.

(3) Likewise we say a type $p(x)$ Kim-forks or Kim-divides over $A$ if it implies a formula that Kim-forks or Kim-divides over $A$, respectively.

(4) We write $a \downarrow_{A}^{K} b$ to denote the assertion that $\operatorname{tp}(a / A b)$ does not Kim-fork over $A$.

In [9], the following definition of Kim-dividing was introduced: $\varphi(x ; a)$ Kimdivides over $A$ if $\left\{\varphi\left(x ; a_{i}\right): i<\omega\right\}$ is inconsistent for some Morley sequence $\left\langle a_{i}\right|$ $i\langle\omega\rangle$ in an $A$-invariant global type with $a=a_{0}$. However, by [9, Theorem 7.7], the definition of Kim-dividing given above is equivalent to the definition of Kimdividing over models in $\mathrm{NSOP}_{1}$ theories. There are known examples even of simple theories where types over sets (even assuming they are boundedly closed) do not have global invariant extensions (see 2]) so the above definition will be the more fruitful one for extending the theory to arbitrary sets.

We remark that if $A$ and $B$ are sets of parameters and $p$ is a partial type over $B$ that does not Kim-fork over $A$, then there is a complete type $q$ over $B$ extending $p$ which also does not Kim-fork over $A$. This does not require existence and follows from the same argument as the analogous statement for non-forking: one simply takes any completion of $p$ together with the negation of all formulas over $B$ which Kim-fork over $A$, which is consistent by compactness. 
The following facts summarize the key properties of Kim-independence that have been established over models:

Fact 2.11. [9, Theorem 3.15] The following are equivalent for the complete theory $T:$

(1) $T$ is $\mathrm{NSOP}_{1}$.

(2) Kim's lemma for Kim-dividing over models: Given any model $M \models T$ and formula $\varphi\left(x ; b_{0}\right), \varphi\left(x ; b_{0}\right)$ Kim-divides over $M$ if and only if for any $\left\langle b_{i}\right.$ : $i<\omega\rangle$ Morley over $M$ in some global $M$-invariant type, $\left\{\varphi\left(x ; b_{i}\right): i<\omega\right\}$ is inconsistent.

(3) Symmetry of Kim independence over models: $a \downarrow_{M}^{K} b$ iff $b \downarrow_{M}^{K} a$ for any $M \models T$.

(4) Independence theorem over models: if $A \downarrow_{M}^{K} B, c \downarrow_{M}^{K} A, c^{\prime} \downarrow_{M}^{K} B$ and $c \equiv_{M} c^{\prime}$ then there is some $c^{\prime \prime} \downarrow_{M}^{K} A B$ such that $c^{\prime \prime} \equiv_{M A} c$ and $c^{\prime \prime} \equiv_{M B} c^{\prime}$.

(5) If $\varphi\left(x ; a_{0}\right)$ does not Kim-divide over $M$ and $\left\langle a_{i}: i<\omega\right\rangle$ is an $M$-indiscernible sequence with $a_{i} \mathfrak{L}_{M}^{K} a_{<i}$ then $\left\{\varphi\left(x ; a_{i}\right): i<\omega\right\}$ is consistent.

Lemma 2.12. Assume $T$ satisfies the existence axiom. Suppose that $I=\left\langle a_{i}\right.$ : $i<\omega\rangle$ is Morley over $A$. Then for any $b^{\prime}$ there are $b_{i}$ such that $a_{0} b^{\prime} \equiv_{A} a_{0} b_{0}$ and $\left\langle a_{i} b_{i} \mid i<\omega\right\rangle$ is Morley over $A$.

Proof. We may stretch $I$ to $\left\langle a_{i}: i<\kappa\right\rangle$ for a sufficiently large $\kappa$. By induction on $i<\kappa$, we will choose $a_{i}^{\prime} b_{i}^{\prime}$ so that

(1) $a_{i}^{\prime} b_{i}^{\prime} \equiv_{A} a_{0} b^{\prime}$ and $a_{\leq i}^{\prime} \equiv_{A} a_{\leq i}$.

(2) $a_{i}^{\prime} \downarrow_{A} a_{<i}^{\prime} b_{<i}^{\prime}$.

(3) $b_{i}^{\prime} \downarrow_{A a_{i}^{\prime}} a_{<i}^{\prime} b_{<i}^{\prime}$ for all $i<\kappa$.

Suppose for some $i<\kappa$, we have chosen $a_{<i}^{\prime} b_{<i}^{\prime}$ satisfying the above requirements. Since $a_{<i}^{\prime} \equiv_{A} a_{<i}$, there is $a_{i}^{\prime}$ such that $a_{<i}^{\prime} a_{i}^{\prime} \equiv_{A} a_{<i} a_{i}$ and therefore $a_{i}^{\prime} \downarrow_{A} a_{<i}^{\prime}$. By extension, we may assume $a_{i}^{\prime} \downarrow_{A} a_{<i}^{\prime} b_{<i}^{\prime}$. By existence and extension, there is $b_{i}^{\prime}$ such that $a_{i}^{\prime} b_{i}^{\prime} \equiv_{A} a_{0} b^{\prime}$ and $b_{i}^{\prime} \downarrow_{A a_{i}^{\prime}} a_{<i}^{\prime} b_{<i}^{\prime}$. The pair $a_{i}^{\prime} b_{i}^{\prime}$ satisfies the requirements and, by induction, completes the construction of $\left\langle a_{i}^{\prime} b_{i}^{\prime}: i<\kappa\right\rangle$.

Note that, by the left-transitivity of non-forking independence, conditions (2) and (3) imply that $a_{i}^{\prime} b_{i}^{\prime} \downarrow_{A} a_{<i}^{\prime} b_{<i}^{\prime}$ for all $i<\kappa$. By applying Erdös-Rado (Fact 2.4), we obtain an $A$-indiscernible sequence $\left\langle a_{i}^{\prime \prime} b_{i}^{\prime \prime}: i<\kappa\right\rangle$ which also satisfies (1)(3). By (1) and an automorphism, we can find $b_{i}$ for all $i<\kappa$ so that $\left\langle a_{i}^{\prime \prime} b_{i}^{\prime \prime}: i<\right.$ $\kappa\rangle \equiv{ }_{A}\left\langle a_{i} b_{i}: i<\kappa\right\rangle$, which gives the desired sequence.

Finally, we observe that the property of Kim-dividing depends only on the set defined by a formula, not on the formula itself.

Corollary 2.13. Assume $T$ satisfies the existence axiom.

(1) Kim-dividing of a formula depends only on the definable set defined by the formula.

(2) If $\varphi_{0}(x, a) \models \varphi_{1}(x, b)$ and $\varphi_{1}(x, b)$ Kim-divides over $A$, then so does $\varphi_{0}(x, a)$.

Proof. (1) The assertion is clear for Kim-forking, so we prove it only for Kimdividing. Let $\models \varphi\left(x, a_{0}\right) \leftrightarrow \psi\left(x, b_{0}\right)$ and assume $\left\{\varphi\left(x, a_{i}\right): i<\omega\right\}$ is inconsistent for some Morley sequence $I=\left\langle a_{i}: i<\omega\right\rangle$ over $A$. By Lemma 2.12, there is 
a Morley sequence $\left\langle b_{i}: i<\omega\right\rangle$ over $A$ such that $\varphi\left(x, a_{i}^{\prime}\right) \leftrightarrow \psi\left(x, b_{i}\right)$ with some $\left\langle a_{i}^{\prime}\right\rangle \equiv_{A} I$, so $\left\{\psi\left(x, b_{i}\right)\right\}_{i}$ is inconsistent. This shows that if $\varphi\left(x ; a_{0}\right)$ Kim-divides over $A$, then $\psi\left(x ; b_{0}\right)$ Kim-divides over $A$, and by symmetry we conclude. (2) follows from Lemma 2.12 as well, by an entirely similar argument.

Clearly, the above corollary remains true if we replace Kim-dividing by Kimforking, even if we do not assume the existence axiom.

Finally, we note that there are many $\mathrm{NSOP}_{1}$ theories for which our results apply. The existence axiom has been proved explicitly in almost every $\mathrm{NSOP}_{1}$ theory that has been studied in detail, and there is no known example of an $\mathrm{NSOP}_{1}$ theory in which existence fails.

Fact 2.14. The following theories are $\mathrm{NSOP}_{1}$ and satisfy the existence axiom:

(1) Any complete theory of $\omega$-free PAC fields. 1, Proposition 3.1] 4, Corollary $6.2]$

(2) The theory $T_{m, n}$ of existentially closed incidence structures omitting the complete incidence structure $K_{m, n}$. [5. Theorem 4.11, Corollary 4.24]

(3) The theory $T_{f e q}^{*}$ of parameterized equivalence relations. [4, Corollary 6.4]

(4) For any language $L$, the model completion of the empty theory $T_{L}^{\emptyset}$. 14 , Corollary 3.13, Proposition 3.17]

(5) The theory ACFG of an algebraically closed field of characteristic $p$ with a predicate naming a generic multiplicative subgroup. [7, Theorem 5.9] [6, Corollary 3.13]

Remark 2.15. Although it does not appear explicitly in the literature, we sketch how existence may be shown for $T_{f e q}^{*}$. Recall that the language contains two unary relations $P$ and $O$, and a ternary relation $E_{x}(y, z)$. The theory $T_{f e q}$ asserts that $P$ and $O$ are disjoint, $E_{x}(y, z)$ implies $x \in P$ and $(y, z) \in O^{2}$, and, for every $p \in P$, $E_{p}(y, z)$ is an equivalence relation on $O$. Given $p \in P$ and $c \in O$, we write $[c]_{E_{p}}$ for the $E_{p}$-class of $c$. The model completion $T_{f e q}^{*}$ is $\aleph_{0}$-categorical, with trivial algebraic closure and elimination of quantifiers, see [4, Subsection 6.3] for further details.

Suppose $\mathbb{M}$ is a monster model of $T_{\text {feq }}^{*}, A \subseteq \mathbb{M}$ is a small set of parameters, and $q \in S(A)$. We can write $q=q\left(x_{0}, \ldots, x_{n-1}, y_{0}, \ldots, y_{m-1}\right)$ where $q \vdash x_{i} \in O$ and $q \vdash y_{j} \in P$ for all $i<n, j<m$. We, moreover, may easily reduce to the case that $q$ implies no equality between an $x_{i}$ or $y_{j}$ and an element of $A$. Define a global type extending $q$ with the following formulas:

- For all $i<n, p \in P(\mathbb{M}) \backslash A, c \in O(\mathbb{M})$, we add $\neg E_{p}\left(x_{i}, c\right)$.

- For all $i<n, p \in P(A)$, and $c \in \mathbb{M}$ such that $[c]_{E_{p}} \cap A=\emptyset$, we put $\neg E_{p}\left(x_{i}, c\right)$.

- For all $j<m$ and $c \in \mathbb{M}$, we add $y_{j} \neq c$.

- For all $j<m, c \in \mathbb{M}$, and $c^{\prime} \in \mathbb{M} \backslash A$, we add $\neg E_{y_{j}}\left(c, c^{\prime}\right)$, unless $c=c^{\prime}$.

It is easy to check that this implies a complete global type and is moreover $A$ invariant, hence does not fork over $A$. As $q$ and $A$ are arbitrary, this shows existence for $T_{f e q}^{*}$.

2.3. Transfer to models. In this subsection, we explain how, from a Morley sequence over $A$, one can find a model $M$ containing $A$ such that the sequence is finitely satisfiable in $M$ and $M$ is independent from the sequence over $A$. This will be a key step in reducing Kim's lemma for Kim-dividing over arbitrary sets to the 
known version of Kim's lemma for Kim-dividing over models. Our proof makes use of notions related to the fundamental order from classical stability theory (see [15]).

\section{Throughout this subsection we assume the existence axiom.}

For $A \subseteq M$ and finite $a$, put

$$
\mathrm{Cl}_{A}(a / M):=\{\varphi(x, y) \in \mathcal{L}(A) \mid \varphi(x, m) \in \operatorname{tp}(a / M) \text { for some } m \in M\} .
$$

Lemma 2.16. Let $I=\left\langle a_{i} \mid i<\omega\right\rangle$ be an A-Morley sequence. Then there is a model $M \supseteq A$ such that $M \downarrow_{A} I, I$ is $M$-indiscernible, and $\operatorname{tp}\left(a_{<k} / M a_{\geq k}\right)$ is an heir extension of $\operatorname{tp}\left(a_{<k} / M\right)$ for any $k<\omega$ (so I is a coheir sequence over $M$ ).

Proof. Consider the following class of models

$$
\mathcal{U}_{0}:=\left\{N \prec \mathbb{M}|A \subseteq N,| N \mid<\bar{\kappa}, I \text { is } N \text {-indiscernible, and } N \downarrow_{A} I\right\},
$$

which is ordered as follows: $N_{1}<N_{2}$ if $N_{1} \prec N_{2}$ and $\mathrm{Cl}_{A}\left(I / N_{1}\right)$ (with object variables $x_{0}, x_{1}, \ldots$ corresponding to $\left.a_{0}, a_{1}, \ldots\right)$ is a proper subset of $\mathrm{Cl}_{A}\left(I / N_{2}\right)$. Due to the existence axiom, there is a model $M^{\prime} \supseteq A$ such that $M^{\prime} \downarrow_{A} I$. Then, by Ramsey's theorem and compactness, there is $I^{\prime} \equiv_{A} I$ such that $I^{\prime}$ is $M^{\prime}$-indiscernible and $M^{\prime} \downarrow_{A} I^{\prime}$ : by Ramsey and compactness, from $I$, we extract an $M^{\prime}$-indiscernible sequence $I^{\prime}=\left\langle a_{i}^{\prime}: i<\omega\right\rangle$. Since $I$ was already $A$-indiscernible, we have $I^{\prime} \equiv_{A} I$. If $M^{\prime} \grave{Z}_{A} I^{\prime}$, then there are $i_{0}<\ldots<i_{n-1}$ and $\varphi\left(x ; a_{i_{0}}^{\prime}, \ldots, a_{i_{n-1}}^{\prime}\right) \in \operatorname{tp}\left(M^{\prime} / A I^{\prime}\right)$ which forks over $A$. But then, as $I^{\prime}$ was extracted from $I$, there are $j_{0}<\ldots<j_{n-1}$ so that $\varphi\left(x ; a_{j_{0}}, \ldots, a_{j_{n-1}}\right) \in \operatorname{tp}\left(M^{\prime} / A I\right)$. Since $a_{i_{0}}^{\prime} \ldots a_{i_{n-1}}^{\prime} \equiv_{A} a_{j_{0}} \ldots a_{j_{n-1}}$, it follows that $\varphi\left(x ; a_{j_{0}}, \ldots, a_{j_{n-1}}\right)$ also forks over $A$, contradicting $M^{\prime} \downarrow_{A} I$. This shows $M^{\prime} \downarrow_{A} I^{\prime}$.

Hence by an $A$-automorphism sending $I^{\prime}$ to $I$, we see that $\mathcal{U}_{0}$ is non-empty. Moreover, any chain in $\mathcal{U}_{0}$ has length at most $|\mathcal{L}(A)|$ so its union is also in $\mathcal{U}_{0}$. Therefore, by Zorn's Lemma there is a maximal element in $\mathcal{U}_{0}$, say $M_{0}$.

Claim. For any $k<l<\omega$, an $\mathcal{L}(A)$-formula $\varphi(\bar{x}, y, \bar{z})$ and $m \in M_{0}$ such that $\models \varphi\left(a_{<k}, m, a_{k} \ldots a_{l}\right)$, there is $m^{\prime} \bar{m}^{\prime \prime} \in M_{0}$ such that $\varphi\left(a_{<k}, m^{\prime}, \bar{m}^{\prime \prime}\right)$ holds.

Proof of Claim. By compactness, clearly there is $I^{\prime}$ such that $I I^{\prime}$ is $M_{0}$-indiscernible and $A$-Morley, and $M_{0} \downarrow_{A} I I^{\prime}$. We can further assume the length of $I$ is sufficiently large. Note that $M_{0} \downarrow_{A I^{\prime}} I$, and, by Fact 2.5 $I^{\prime} \downarrow_{A} I$. Thus, by lefttransitivity, $M_{0} I^{\prime} \downarrow_{A} I$ holds. Now, again by existence, there is $M_{0}^{\prime} \supseteq M_{0} I^{\prime}$ such that $M_{0}^{\prime} \downarrow_{M_{0} I^{\prime}} I$. Then since $I$ is $M_{0} I^{\prime}$-indiscernible, we can, as above, apply Ramsey and compactness to assume $I$ (with its original length $\omega$ ) is $M_{0^{-}}^{\prime}$ indiscernible. Due to left-transitivity we have $M_{0}^{\prime} \downarrow_{A} I$, so by the maximality of $M_{0}$, we have $\mathrm{Cl}_{A}\left(I / M_{0}\right)=\mathrm{Cl}_{A}\left(I / M_{0}^{\prime}\right)$. Moreover, there are $a_{k}^{\prime} \ldots a_{l}^{\prime} \in I^{\prime}$ such that $a_{<k} a_{k} \ldots a_{l} \equiv_{M_{0}} a_{<k} a_{k}^{\prime} \ldots a_{l}^{\prime}$. Then $\models \varphi\left(a_{<k}, m, a_{k}^{\prime} \ldots a_{l}^{\prime}\right)$, so $\varphi(\bar{x} ; y, \bar{z}) \in$ $\mathrm{Cl}_{A}\left(I / M_{0}^{\prime}\right)=\mathrm{Cl}_{A}\left(I / M_{0}\right)$, which gives the claim.

As we considered formulas over $A$ (not over $M_{0}$ ), $M_{0}$ is not yet a desired model. Now we iterate this argument to obtain an elementary chain $\left\{M_{i} \mid i<\omega\right\}$ such that $M_{n+1}$ is a maximal element in

$$
\mathcal{U}_{n+1}:=\left\{N \mid M_{n} \prec N \text {, and } I \text { is } N \text {-indiscernible, and } N \downarrow_{A} I\right\},
$$

ordered by: $N_{1}<N_{2}$ if $N_{1} \prec N_{2}$ and $\mathrm{Cl}_{M_{n}}\left(I / N_{1}\right) \subsetneq \mathrm{Cl}_{M_{n}}\left(I / N_{2}\right)$. Then by the same argument as above, the same Claim (except $\varphi$ is assumed to be an $\mathcal{L}\left(M_{n}\right)$ formula, $m \in M_{n+1}$ and $\left.m^{\prime} \bar{m}^{\prime \prime} \in M_{n+1}\right)$ holds. Therefore we put $M:=\bigcup_{n<\omega} M_{n}$, which is a desired model. 
By choosing a maximal element in $\mathcal{U}_{0}$ in the proof of Lemma 2.16 suitably, indeed we have proved the following.

Lemma 2.17. Let I be an A-Morley sequence. Then there is a model $M \supseteq A$ such that $M \downarrow_{A} I$ and $I$ is a coheir sequence over $M$. Moreover, if we are given $M_{0} \supseteq A$ such that $M_{0} \downarrow_{A} I$ and $I$ is $M_{0}$-indiscernible, then there is a model $M \succ M_{0}$ such that $M \downarrow_{A} I$ and $I$ is a coheir sequence over $M$.

\section{Kim's LEMma OVER ARBITRARY SETS}

Until the end of Section 5 we assume $T$ has NSOP $_{1}$ and satisfies existence for forking independence.

For an ordinal $\alpha$, let the language $L_{s, \alpha}$ be $\left\langle\unlhd, \wedge,<_{l e x},\left(P_{\beta}\right)_{\beta \leq \alpha}\right\rangle$. We may view a tree with $\alpha$ levels as an $L_{s, \alpha}$-structure by interpreting $\unlhd$ as the tree partial order, $\wedge$ as the binary meet function, $<_{l e x}$ as the lexicographic order, and $P_{\beta}$ interpreted to define level $\beta$.

Recall the modeling property:

Definition 3.1. Suppose $I$ is an $L^{\prime}$-structure, where $L^{\prime}$ is some language.

(1) We say $\left(a_{i}: i \in I\right)$ is a set of $I$-indexed indiscernibles over $A$ if whenever $\left(s_{0}, \ldots, s_{n-1}\right),\left(t_{0}, \ldots, t_{n-1}\right)$ are tuples from $I$ with

$$
\operatorname{qftp}_{L^{\prime}}\left(s_{0}, \ldots, s_{n-1}\right)=\operatorname{qftp}_{L^{\prime}}\left(t_{0}, \ldots, t_{n-1}\right),
$$

then we have

$$
\operatorname{tp}\left(a_{s_{0}}, \ldots, a_{s_{n-1}} / A\right)=\operatorname{tp}\left(a_{t_{0}}, \ldots, a_{t_{n-1}} / A\right) .
$$

(2) In the case that $L^{\prime}=L_{s, \alpha}$ for some $\alpha$, we say that an $I$-indexed indiscernible is $s$-indiscernible. As the only $L_{s, \alpha}$-structures we will consider will be trees, we will often refer $I$-indexed indiscernibles in this case as s-indiscernible trees.

(3) We say that $I$-indexed indiscernibles have the modeling property if, given any $\left(a_{i}: i \in I\right)$ from $\mathbb{M}$ and any $A$, there is an $I$-indexed indiscernible $\left(b_{i}: i \in I\right)$ over $A$ in $\mathbb{M}$ locally based on $\left(a_{i}: i \in I\right)$ over $A$ - i.e., given any finite set of formulas $\Delta$ from $\mathcal{L}(A)$ and a finite tuple $\left(t_{0}, \ldots, t_{n-1}\right)$ from $I$, there is a tuple $\left(s_{0}, \ldots, s_{n-1}\right)$ from $I$ so that

$$
\operatorname{qftp}_{L^{\prime}}\left(t_{0}, \ldots, t_{n-1}\right)=\mathrm{qftp}_{L^{\prime}}\left(s_{0}, \ldots, s_{n-1}\right)
$$

and also

$$
\operatorname{tp}_{\Delta}\left(b_{t_{0}}, \ldots, b_{t_{n-1}}\right)=\operatorname{tp}_{\Delta}\left(a_{s_{0}}, \ldots, a_{s_{n-1}}\right) .
$$

Fact 3.2. [12, Theorem 4.3] Let $I_{s}$ denote the $L_{s, \omega}$-structure $\left(\omega^{<\omega}, \unlhd,<_{l e x}, \wedge,\left(P_{\alpha}\right)_{\alpha<\omega}\right)$ with all symbols being given their intended interpretations and each $P_{\alpha}$ naming the elements of the tree at level $\alpha$. Then $I_{s}$-indexed indiscernibles have the modeling property.

Remark 3.3. It follows by compactness that for any cardinal $\kappa$, if $\left(a_{\eta}\right)_{\eta \in \omega<\kappa}$ is a collection of tuples, then there is an $s$-indiscernible tree $\left(b_{\eta}\right)_{\eta \in \omega<\kappa}$ locally based on $\left(a_{\eta}\right)_{\eta \in \omega<\kappa}$. For arbitrary $\kappa$, one considers the partial type $\Gamma\left(x_{\eta}: \eta \in \omega^{<\kappa}\right)$ consisting of formulas naturally asserting that $\left(x_{\eta}\right)_{\eta \in \omega<\kappa}$ is $s$-indiscernible, together with every formula of the form $\varphi\left(\bar{x}_{\bar{\eta}}\right)$ where $\models \varphi\left(\bar{a}_{\bar{\nu}}\right)$ for all tuples $\bar{\nu}$ from $\omega^{<\kappa}$ realizing $\operatorname{qftp}_{L_{s, \kappa}}(\bar{\eta})$. Fact 3.2 may be used to show any finite subset is satisfiable and a realization will be the desired $s$-indiscernible tree. 
Lemma 3.4. Suppose $A$ is a set of parameters, $I=\left\langle a_{i}: i<\omega\right\rangle$ and $J=\left\langle b_{i}: i<\omega\right\rangle$ are $A$-indiscernible sequences with $a_{0}=b_{0}$ and $b_{>0} \downarrow_{A}^{d} b_{0}$. Then there is a tree $\left(c_{\eta}\right)_{\eta \in \omega<\omega}$ satisfying the following properties:

(1) For all $\eta \in \omega^{<\omega},\left(c_{\eta \frown\langle i\rangle}\right)_{i<\omega} \equiv_{A} I$.

(2) For all $\eta \in \omega^{<\omega},\left(c_{\eta}, c_{\eta \mid l(\eta)-1}, \ldots, c_{\emptyset}\right) \equiv_{A}\left(b_{0}, b_{1}, \ldots, b_{l(\eta)}\right)$.

(3) $\left(c_{\eta}\right)_{\eta \in \omega<\omega}$ is s-indiscernible over $A$.

Proof. By induction on $n$, we will construct a tree $\left(c_{\eta}\right)_{\eta \in \omega \leq n}$ so that

(1) For all $\eta \in \omega^{<n},\left(c_{\eta \frown\langle i\rangle}\right)_{i<\omega} \equiv_{A} I$.

(2) For all $\eta \in \omega^{n},\left(c_{\eta}, c_{\eta \mid(n-1)}, \ldots, c_{\eta \mid 0}\right) \equiv_{A}\left(b_{0}, \ldots, b_{n}\right)$.

For $n=0$, we may set $c_{\emptyset}=b_{0}$, which trivially satisfies the requirements. Now suppose we are given $\left(c_{\eta}\right)_{\eta \in \omega \leq n}$. By the indiscernibility of $J$ and (2), we have $\left(c_{0^{n}}, c_{0^{n-1}}, \ldots, c_{\emptyset}\right) \equiv_{A}\left(b_{1}, \ldots, b_{n+1}\right)$ so we may choose $c_{*}$ so that $c_{*}\left(c_{0^{n}}, c_{0^{n-1}}, \ldots, c_{\emptyset}\right) \equiv_{A}$ $b_{0}\left(b_{1}, \ldots, b_{n+1}\right)$. By invariance and $b_{>0} \downarrow_{A}^{d} b_{0}$, we have $c_{0^{n}} c_{0^{n-1}} \ldots c_{\emptyset} \downarrow_{A}^{d} c_{*}$. Let $I_{*}=\left\langle c_{*, i}: i<\omega\right\rangle$ be a sequence with $I_{*} \equiv_{A} I$ and $c_{*, 0}=c_{*}$. Since $c_{0^{n}} c_{0^{n-1}} \ldots c_{\emptyset} \downarrow_{A}^{d} c_{*}$, we may assume $I_{*}$ is $A c_{0^{n}} c_{0^{n-1}} \ldots c_{\emptyset}$-indiscernible and, therefore, that for all $i<\omega$,

$$
c_{*, i} c_{0^{n}} \ldots c_{\emptyset} \equiv_{A} b_{0} b_{1} \ldots b_{n+1} .
$$

Now we define a tree $\left(c_{\eta}\right)_{\eta \in \omega \leq n+1}$ by placing a copy of $I$ on top of each node of level $n$ in $\left(c_{\eta}\right)_{\eta \in \omega \leq n}$. More precisely, using (2), we may choose, for each $\eta \in \omega^{n}$, an automorphism $\sigma_{\eta} \in \operatorname{Aut}(\mathbb{M} / A)$ so that $\sigma_{\eta}\left(c_{0^{n}} c_{0^{n-1}} \ldots c_{\emptyset}\right)=c_{\eta} c_{\eta \mid(n-1)} \ldots c_{\emptyset}$. We may take $\sigma_{0^{n}}=\operatorname{id}_{\mathbb{M}}$. Now to define $\left(c_{\eta}\right)_{\eta \in \omega \leq n+1}$, we put, for each $\eta \in \omega^{n}$ and $i<\omega, c_{\eta \frown\langle i\rangle}=\sigma_{\eta}\left(c_{*, i}\right)$. By induction and the construction, the tree constructed this way satisfies (1). Moreover, for all $\eta \in \omega^{n}$,

$$
c_{\eta \frown\langle i\rangle} c_{\eta} \ldots c_{\emptyset} \equiv_{A} c_{*, i} c_{0^{n}} \ldots c_{\emptyset} \equiv_{A} b_{0} b_{1} \ldots b_{n+1},
$$

so this tree satisfies (2) as well.

This completes the inductive construction of $\left(c_{\eta}\right)_{\eta \in \omega}<\omega$. By applying Fact 3.2 to extract $\left(c_{\eta}^{\prime}\right)_{\eta \in \omega<\omega} s$-indiscernible over $A$ and locally based on $\left(c_{\eta}\right)_{\eta \in \omega<\omega}$, we obtain the desired tree.

For the following proof, recall that we say $\left(a_{i, j}\right)_{i<\kappa, j<\lambda}$ is a mutually indiscernible array over $A$ if, for each $i<\kappa$, the sequence $\bar{a}_{i}=\left\langle a_{i, j}: j\langle\lambda\rangle\right.$ is indiscernible over $A \bar{a}_{\neq i}$.

Theorem 3.5. T satisfies Kim's lemma for Kim-dividing over an arbitrary set A: a formula $\varphi(x, a)$ Kim-divides over $A$ with respect to some Morley sequence in $\operatorname{tp}(a / A)$ iff it Kim-divides over $A$ with respect to any such sequence.

Proof. Towards contradiction, assume we are given $\varphi(x ; a)$ and Morley sequences $I=\left\langle a_{i}: i<\omega\right\rangle$ and $J=\left\langle b_{i}: i<\omega\right\rangle$ over $A$ both of which are in $\operatorname{tp}(a / A)$ and such that $\left\{\varphi\left(x ; a_{i}\right): i<\omega\right\}$ is consistent and $\left\{\varphi\left(x ; b_{i}\right): i<\omega\right\}$ is inconsistent.

As $J$ is a Morley sequence over $A$, we have $b_{>0} \downarrow_{A} b_{0}$ so, by Lemma 3.4 we can find a tree $\left(c_{\eta}\right)_{\eta \in \omega<\omega}$ which satisfies:

(1) For all $\eta \in \omega^{<\omega},\left(c_{\eta \frown\langle i\rangle}\right)_{i<\omega} \equiv_{A} I$.

(2) For all $\eta \in \omega^{<\omega},\left(c_{\eta}, c_{\eta \mid l(\eta)-1}, \ldots, c_{\emptyset}\right) \equiv_{A}\left(b_{0}, b_{1}, \ldots, b_{l(\eta)}\right)$.

(3) $\left(c_{\eta}\right)_{\eta \in \omega<\omega}$ is $s$-indiscernible over $A$.

Define an array $\left(d_{i, j}\right)_{i, j<\omega}$ by $d_{i j}=c_{0^{i} \frown(j+1)}$. By $s$-indiscernibility, $\left(d_{i, j}\right)_{i, j<\omega}$ is mutually indiscernible and, moreover, $\bar{d}_{i} \equiv_{A} I$ for all $i<\omega$. By compactness 
applied to $\left(d_{i, j}\right)_{i, j<\omega}$ and $\left(c_{0^{i+1}}\right)_{i<\omega}$, for $\kappa$ large enough, we can find an $A$-mutually indiscernible array $\left(e_{i, j}\right)_{i<\kappa, j<\omega}$ and an $A$-indiscernible sequence $\left(e_{i}^{\prime}\right)_{i<\kappa}$ having the same EM-type over $A$ as $J$ (with the reversed order) such that $\bar{e}_{i} \equiv_{A} I$ and $e_{i}^{\prime} \equiv_{A e_{<i} e_{<i}^{\prime}} e_{i, 0}$ for all $i<\kappa$. By Fact 2.4 we may assume $\kappa=\omega$ and $\left(\bar{e}_{i}\right)_{i<\omega}$ is $A$-indiscernible.

By applying Lemma 2.17, we can find a model $M \supseteq A$ such that $\bar{e}_{0}$ is a Morley sequence over $M$ and $M \downarrow_{A} \bar{e}_{0}$. Then because $\left(\bar{e}_{i}\right)_{i<\omega}$ is $A$-indiscernible, we may, moreover, assume $M$ has been chosen so that $\left(\bar{e}_{i}\right)_{i<\omega}$ is $M$-indiscernible. Let $\lambda$ be any cardinal larger than $2^{|\mathcal{L}|+|M|}$ and apply compactness to stretch the array to $\left(e_{i, j}\right)_{i<\omega, j<\lambda}$, preserving $A$-mutual indiscernibility and the $M$-indiscernibility of $\left(\bar{e}_{i}\right)_{i<\omega}$.

Now by induction, we will find $\alpha_{n}<\lambda$ so that $e_{n, \alpha_{n}} \downarrow_{M}^{K} e_{<n, \alpha_{<n}}$ for all $n<\omega$. Suppose we have succeeded in finding $\left(\alpha_{m}\right)_{m<n}$. Then by the pigeonhole principle and the choice of $\lambda$, there is an infinite subsequence $I_{n}$ of $\bar{e}_{n}$ so that every tuple of $I_{n}$ has the same type over $M e_{<n, \alpha_{<n}}$. Let $\alpha_{n}<\lambda$ be least such that $e_{n, \alpha_{n}} \in I_{n}$. As $I_{n}$ is a subsequence of a Morley sequence over $M, I_{n}$ is also Morley over $M$ and hence, by Kim's lemma for Kim-dividing, we have $e_{<n, \alpha_{<n}} \downarrow_{M}^{K} e_{n, \alpha_{n}}$ which, by symmetry, is what we need to complete the induction.

We claim that $\left\{\varphi\left(x ; e_{n, \alpha_{n}}\right): n<\omega\right\}$ is consistent. By compactness, it suffices to show that $\left\{\varphi\left(x ; e_{n, \alpha_{n}}\right): n<N\right\}$ does not Kim-divide over $M$ for any $N$. This is true for $N=1$ by Kim's lemma, since $\left\{\varphi\left(x ; e_{0, j}\right): j<\lambda\right\}$ is consistent and $\bar{e}_{0}$ is a Morley sequence over $M$. Assuming we have shown it for $N$, we can choose $c_{N} \models\left\{\varphi\left(x ; e_{n, \alpha_{n}}\right): n<N\right\}$ with $c_{N} \downarrow_{M}^{K} e_{<N, \alpha_{<N}}$. Additionally, since $e_{0, \alpha_{0}} \equiv_{M} e_{N, \alpha_{N}}$, we can choose $c$ so that $c_{N} e_{0, \alpha_{0}} \equiv_{M} c e_{N, \alpha_{N}}$, from which it follows $c \downarrow_{M}^{K} e_{N, \alpha_{N}}$ by invariance. Applying the independence theorem over $M$, we find $c_{N+1} \models \operatorname{tp}\left(c_{N} / M e_{<N, \alpha_{<N}}\right) \cup \operatorname{tp}\left(c / M e_{N, \alpha_{N}}\right)$. In particular, we have $c_{N+1} \models\left\{\varphi\left(x ; e_{n, \alpha_{n}}\right): n<N+1\right\}$, and therefore $\left\{\varphi\left(x ; e_{n, \alpha_{n}}\right): n<N+1\right\}$ does not Kim-divide, completing the induction.

By mutual indiscernibility over $A$, we also have $\left\{\varphi\left(x ; e_{i, 0}\right): i<\omega\right\}$ is consistent. By $\mathrm{NSOP}_{1}$ and Fact 2.9, it follows that $\left\{\varphi\left(x ; e_{i}^{\prime}\right): i<\omega\right\}$ is also consistent. But this entails that $\left\{\varphi\left(x ; b_{i}\right): i<\omega\right\}$ is consistent, a contradiction.

Remark 3.6. An alternative conclusion to the argument may be given, less elementary but with fewer moving parts. Starting from the definition of the array $\left(d_{i, j}\right)_{i, j<\omega}$ by $d_{i j}=c_{0^{i} \frown(j+1)}$, we let $\left(\bar{d}_{i}^{\prime}\right)_{i<\omega}$ be an $A$-indiscernible sequence locally based on $\left(\bar{d}_{i}\right)_{i<\omega}$. By Lemma 2.17 there is a model $M \supseteq A$ so that $M \downarrow_{A}^{f} \bar{d}_{0}^{\prime}$ and $\bar{d}_{0}^{\prime}$ is a coheir sequence over $M$, and, as above, we may assume $\left(d_{i}^{\prime}\right)_{i<\omega}$ is $M$ indiscernible. Finally, let $\left(e_{i, j}\right)_{i, j<\omega}$ be an array that is mutually indiscernible over $M$ (see, e.g., 3, Lemma 1.2]) and locally based on $\left(d_{i, j}^{\prime}\right)_{i, j<\omega}$. Notice that for all $f: \omega \rightarrow \omega$, because $\left(d_{i, j}\right)_{i, j<\omega}$ and hence $\left(d_{i, j}^{\prime}\right)_{i, j<\omega}$ are mutually indiscernible over $A$, we have

$$
\left(e_{i, f(i)}\right)_{i<\omega} \equiv_{A}\left(d_{i, f(i)}^{\prime}\right)_{i<\omega} \equiv_{A}\left(d_{i, 0}^{\prime}\right)_{i<\omega} .
$$

Note that for all $i,\left\{\varphi\left(x ; e_{i, j}\right): j<\omega\right\}$ is consistent because $\left\{\varphi\left(x ; a_{j}\right): j<\omega\right\}$ is consistent and $\bar{e}_{i}$ is a coheir sequence over $M$, so $\varphi\left(x ; e_{i, 0}\right)$ does not Kimdivide over $M$. Moreover, by mutual indiscernibility, $\bar{e}_{i}$ is $M \bar{e}_{<i}$-indiscernible so, in particular, $e_{i, 0} \downarrow_{M}^{K} e_{<i, 0}$. This shows $\left\{\varphi\left(x ; e_{i, 0}\right): i<\omega\right\}$ is consistent by Fact $2.11(5)$. Because the indiscernible sequence $\left(d_{i, 0}^{\prime}\right)_{i<\omega}$ is locally based on 
$\left(d_{i, 0}\right)_{i<\omega}=\left(c_{0^{i} \frown 1}\right)_{i<\omega},\left\{\varphi\left(x ; e_{i, 0}\right): i<\omega\right\}$ is consistent if and only if $\left\{\varphi\left(x ; c_{0^{i} \frown 1}\right):\right.$ $i<\omega\}$ is not $k$-inconsistent for any $k$. So for any $k$, we can find $i(0)<\ldots<$ $i(k-1)$ so that $\left\{\varphi\left(x ; c_{i(j)}\right): j<k\right\}$ is consistent. By $s$-indiscernibility we have $\left.c_{0^{i(j)+1}} \equiv_{A\left(c_{0^{i}(l)+1} c_{0^{i}(l)}-1\right.}\right)_{l<j} c_{0^{i(j)}-1}$ for all $j<k$ and $\left\{\varphi\left(x ; c_{0^{i(j)}}\right): j<k\right\}$ is $m$ inconsistent for some $m$ (which does not depend on $k$ ), by our assumption that $\left\{\varphi\left(x ; b_{i}\right): i<\omega\right\}$ is inconsistent. As $k$ is arbitrary, we obtain $\mathrm{SOP}_{1}$ from Fact 2.9 and compactness.

Corollary 3.7. Suppose $T$ satisfies existence. Then $T$ is $N S O P_{1}$ iff $T$ satisfies Kim's Lemma over arbitrary sets (i.e. the conclusion of Theorem [3.5).

Proof. This follows by Theorem [3.5] and the fact that Kim's Lemma for Kimdividing over models implies (and is equivalent to) $\mathrm{NSOP}_{1}$ ([9, Theorem 3.15]).

\section{Symmetry}

Recall in this section we assume $T$ has $\mathrm{NSOP}_{1}$ and satisfies existence for forking independence.

From the Kim's lemma for Kim-dividing we conclude:

Proposition 4.1. (Kim-forking $=$ Kim-dividing) For any A, if $\varphi(x ; b)$ Kim-forks over $A$ then $\varphi(x ; b)$ Kim-divides over $A$.

Proof. Suppose $\varphi(x ; b) \vdash \bigvee_{j<k} \psi_{j}\left(x ; c^{j}\right)$, where each $\psi_{j}\left(x ; c^{j}\right)$ Kim-divides over $A$. Let $\left(b_{i}, c_{i}^{0}, \ldots, c_{i}^{k-1}\right)_{i<\omega}$ be a Morley sequence in $\operatorname{tp}\left(b, c^{0}, \ldots, c^{k-1} / A\right)$. Since $\left(b_{i}\right)_{i}$ is a Morley sequence in $\operatorname{tp}(b / A)$, to get that $\varphi(x ; b)$ Kim-divides over $A$ it is enough to show that $\left\{\varphi\left(x ; b_{i}\right): i<\omega\right\}$ is inconsistent. If not, then there is some $a \models\left\{\varphi\left(x ; b_{i}\right): i<\omega\right\}$. Then, by the pigeonhole principle, we get that, for some $j<k, a$ realizes $\psi_{j}\left(x ; c_{i}^{j}\right)$, for infinitely many $i$ 's. As $\left(c_{i}^{j}\right)_{i<\omega}$ is a Morley sequence in $\operatorname{tp}\left(c^{j} / A\right)$, it follows from the Kim's lemma that $\psi_{j}\left(x ; c_{i}^{j}\right)$, does not Kim-divide over $A$, a contradiction.

Below, we conclude that, under our assumptions, Kim-dividing (=Kim-forking) satisfies symmetry, by modifying the notion of a Morley tree and the proof of symmetry from [9]. As the argument is essentially the same, we only give a sketch. We briefly recall the notation from $[9$.

Recall the language $L_{s, \alpha}$ and its interpretation in trees were introduced at the beginning of Section 3, Our trees will be understood to be an $L_{s, \alpha}$-structure for some appropriate $\alpha$. We recall the definition of a class of trees $\mathcal{T}_{\alpha}$ below:

Definition 4.2. Suppose $\alpha$ is an ordinal. We define $\mathcal{T}_{\alpha}$ to be the set of functions $f$ such that

- $\operatorname{dom}(f)$ is an end-segment of $\alpha$ of the form $[\beta, \alpha)$ for $\beta$ equal to 0 or a successor ordinal. If $\alpha$ is a successor, we allow $\beta=\alpha$, i.e. $\operatorname{dom}(f)=\emptyset$.

- $\operatorname{ran}(f) \subseteq \omega$.

- finite support: the set $\{\gamma \in \operatorname{dom}(f): f(\gamma) \neq 0\}$ is finite.

We interpret $\mathcal{T}_{\alpha}$ as an $L_{s, \alpha}$-structure by defining

- $f \unlhd g$ if and only if $f \subseteq g$. Write $f \perp g$ if $\neg(f \unlhd g)$ and $\neg(g \unlhd f)$.

- $f \wedge g=\left.f\right|_{[\beta, \alpha)}=\left.g\right|_{[\beta, \alpha)}$ where $\beta=\min \left\{\gamma:\left.f\right|_{[\gamma, \alpha)}=\left.g\right|_{[\gamma, \alpha)}\right\}$, if non-empty (note that $\beta$ will not be a limit, by finite support). Define $f \wedge g$ to be the empty function if this set is empty (note that this cannot occur if $\alpha$ is a limit). 
- $f<_{l e x} g$ if and only if $f \triangleleft g$ or, $f \perp g$ with $\operatorname{dom}(f \wedge g)=[\gamma+1, \alpha)$ and $f(\gamma)<g(\gamma)$

- For all $\beta \leq \alpha, P_{\beta}=\left\{f \in \mathcal{T}_{\alpha}: \operatorname{dom}(f)=[\beta, \alpha)\right\}$.

Definition 4.3. Suppose $\alpha$ is an ordinal.

(1) (Restriction) If $w \subseteq \alpha$, the restriction of $\mathcal{T}_{\alpha}$ to the set of levels $w$ is given by

$\mathcal{T}_{\alpha}\left\lceil w=\left\{\eta \in \mathcal{T}_{\alpha}: \min (\operatorname{dom}(\eta)) \in w\right.\right.$ and $\left.\beta \in \operatorname{dom}(\eta) \backslash w \Longrightarrow \eta(\beta)=0\right\}$.

(2) (Concatenation) If $\eta \in \mathcal{T}_{\alpha}, \operatorname{dom}(\eta)=[\beta+1, \alpha)$, and $i<\omega$, let $\eta \frown\langle i\rangle$ denote the function $\eta \cup\{(\beta, i)\}$. We define $\langle i\rangle \frown \eta \in \mathcal{T}_{\alpha+1}$ to be $\eta \cup\{(\alpha, i)\}$. We write $\langle i\rangle$ for $\emptyset \frown\langle i\rangle$.

(3) (Canonical inclusions) If $\alpha<\beta$, we define the map $\iota_{\alpha \beta}: \mathcal{T}_{\alpha} \rightarrow \mathcal{T}_{\beta}$ by $\iota_{\alpha \beta}(f)=f \cup\{(\gamma, 0): \gamma \in \beta \backslash \alpha\}$.

(4) (The all 0's path) If $\beta<\alpha$, then $\zeta_{\beta}$ denotes the function with $\operatorname{dom}\left(\zeta_{\beta}\right)=$ $[\beta, \alpha)$ and $\zeta_{\beta}(\gamma)=0$ for all $\gamma \in[\beta, \alpha)$. This defines an element of $\mathcal{T}_{\alpha}$ if and only if $\beta \in\{\gamma \in \alpha \mid \gamma$ is not limit $\}=:[\alpha]$.

Definition 4.4. Suppose $\left(a_{\eta}\right)_{\eta \in \mathcal{T}_{\alpha}}$ is a tree of tuples, and $C$ is a set of parameters.

(1) We say $\left(a_{\eta}\right)_{\eta \in \mathcal{T}_{\alpha}}$ is weakly spread out over $C$ if for all $\eta \in \mathcal{T}_{\alpha}$ with $\operatorname{dom}(\eta)=$ $[\beta+1, \alpha)$ for some $\beta<\alpha,\left(a_{\unrhd \eta \frown\langle i\rangle}\right)_{i<\omega}$ is a Morley sequence in $\operatorname{tp}\left(a_{\unrhd \eta}\langle\langle 0\rangle / C)\right.$.

(2) Suppose $\left(a_{\eta}\right)_{\eta \in \mathcal{T}_{\alpha}}$ is a tree which is weakly spread out and $s$-indiscernible over $C$ and for all $w, v \in[\alpha]^{<\omega}$ with $|w|=|v|$,

$$
\left(a_{\eta}\right)_{\eta \in \mathcal{T}_{\alpha} \mid w} \equiv_{C}\left(a_{\eta}\right)_{\eta \in \mathcal{T}_{\alpha} \mid v}
$$

then we say $\left(a_{\eta}\right)_{\eta \in \mathcal{T}_{\alpha}}$ is a weakly Morley tree over $C$.

(3) A weak tree Morley sequence over $C$ is a $C$-indiscernible sequence of the form $\left(a_{\zeta_{\beta}}\right)_{\beta \in[\alpha]}$ for some weakly Morley tree $\left(a_{\eta}\right)_{\eta \in \mathcal{T}_{\alpha}}$ over $C$.

Proposition 4.5. If $a \downarrow_{A}^{K} b$ and $I=\left(b_{i}\right)_{i<\omega}$ is a Morley sequence in $\operatorname{tp}(b / A)$ with $b=b_{0}$, then there is $a^{\prime} \equiv_{A b}$ a such that $a^{\prime} \downarrow_{A}^{K} I$ and $I$ is Aa'-indiscernible.

Proof. Extend $I$ to a sufficiently long Morley sequence $\left(b_{i}\right)_{i<\kappa}$ in $\operatorname{tp}(b / A)$. As $a \downarrow_{A}^{K} b$, we may assume that $b \equiv_{A a} b_{i}$ for all $i<\kappa$ (by moving $a$ over $A b$ ). Then, by Fact 2.4 find an $A a$ indiscernible sequence $\left(b_{j}^{\prime}\right)_{j<\omega}$ based on $\left(b_{i}\right)_{i<\kappa}$ over $A a$. Let $a^{\prime}$ to be the image of $a$ under an automorphism over $A$ sending $\left(b_{j}^{\prime}\right)_{j<\omega}$ to $I$. Then $I$ is $A a^{\prime}$ indiscernible. Now, it is enough to check that for all $n<\omega$, we have that $a^{\prime} \downarrow_{A}^{K} b_{<n}$. But this follows from the indiscernibility and the Kim's lemma, as $\left(b_{k n}, b_{k n+1}, \ldots, b_{k n+n-1}\right)_{k<\omega}$ is a Morley sequence in $\operatorname{tp}\left(b_{<n} / A\right)$ (by left-transitivity of forking independence).

Now, we modify the proof of Lemma 5.11 from 9 , to get:

Lemma 4.6. If $a \downarrow_{A}^{K} b$, then for any ordinal $\alpha \geq 1$, there is a weakly spread out s-indiscernible tree $\left(c_{\eta}\right)_{\eta \in \mathcal{T}_{\alpha}}$ over $A$ such that if $\eta \triangleleft \nu$ and $\operatorname{dom}(\nu)=\alpha$, then $c_{\eta} c_{\nu} \equiv_{A} a b$.

Proof. We will argue by induction on $\alpha$. Suppose $\alpha=1$. Assume $a \downarrow_{A}^{K} b$. Then, by Proposition 4.5. we can choose a Morley sequence in $\operatorname{tp}(b / A)$ which is $A a$ indiscernible. Put $c_{\emptyset}^{1}=a$ and $c_{\langle i\rangle}^{1}=b_{i}$. Then $\left(c_{\eta}^{1}\right)_{\eta \in \mathcal{T}_{1}}$ satisfies the requirements. 
For the successor step, suppose for some $\alpha$ that we have constructed $\left(c_{\eta}^{\beta}\right)_{\eta \in \mathcal{T}_{\beta}}$ for $1 \leq \beta \leq \alpha$ such that, if $\gamma<\beta \leq \alpha$ and $\eta \in \mathcal{T}_{\gamma}$, then $c_{\eta}^{\gamma}=c_{\iota_{\gamma \beta}(\eta)}^{\beta}$. Assume first that $\alpha$ is a successor ordinal. By weak spread-outness, we know that $\left(c_{\unrhd\langle i\rangle}^{\alpha}\right)_{i<\omega}$ is a Morley sequence over $A$, which is, by $s$-indiscernibility over $A, A c_{\emptyset}^{\alpha}$-indiscernible. So $c_{\emptyset}^{\alpha} \downarrow_{A}^{K}\left(c_{\unrhd\langle i\rangle}^{\alpha}\right)_{i<\omega}$. By extension for $\bigsqcup^{K}$, we may find $c^{\prime} \equiv_{A\left(c_{\unrhd\langle i\rangle}^{\alpha}\right)_{i<\omega}} c_{\emptyset}^{\alpha}$ such that

$$
c^{\prime}{\underset{A}{\downarrow}}_{\downarrow}^{K}\left(c_{\eta}^{\alpha}\right)_{\eta \in \mathcal{T}_{\alpha}} .
$$

Let $\left(\left(c_{\eta, i}^{\alpha}\right)_{\eta \in \mathcal{T}_{\alpha}}\right)_{i<\omega}$ be a Morley sequence in $\operatorname{tp}\left(\left(c_{\eta}^{\alpha}\right)_{\eta \in \mathcal{T}_{\alpha}} / A\right)$ with $c_{\eta, 0}^{\alpha}=c_{\eta}^{\alpha}$ for all $\eta \in \mathcal{T}_{\alpha}$. By Proposition 4.5. we can find $c^{\prime \prime} \equiv_{A\left(c_{\eta}^{\alpha}\right)_{\eta \in \mathcal{T}_{\alpha}}} c^{\prime}$ such that $c^{\prime \prime} \bigsqcup_{A}^{K}\left(c_{\eta, i}^{\alpha}\right)_{\eta \in \mathcal{T}_{\alpha}, i<\omega}$ and $\left(\left(c_{\eta, i}^{\alpha}\right)_{\eta \in \mathcal{T}_{\alpha}}\right)_{i<\omega}$ is $A c^{\prime \prime}$-indiscernible.

Define a new tree $\left(d_{\eta}\right)_{\eta \in \mathcal{T}_{\alpha+1}}$ by setting $d_{\emptyset}=c^{\prime \prime}$ and $d_{\eta \cup\{(\alpha, i)\}}=c_{\eta, i}^{\alpha}$ for all $\eta \in \mathcal{T}_{\alpha}$. Then let $\left(c_{\eta}^{\alpha+1}\right)_{\eta \in \mathcal{T}_{\alpha+1}}$ be a tree $s$-indiscernible over $A$ locally based on $\left(d_{\eta}\right)_{\eta \in \mathcal{T}_{\alpha}}$. By an automorphism, we may assume that $c_{\iota_{\alpha \alpha+1}^{\alpha+1}(\eta)}^{\alpha+1}=c_{\eta}^{\alpha}$ for all $\eta \in \mathcal{T}_{\alpha}$. This satisfies our requirements.

If $\alpha$ is a limit ordinal, then, in order to repeat the above argument, find by compactness $c$ which is $\downarrow^{K}$-independent from $\left(c_{\eta}^{\alpha}\right)_{\eta \in \mathcal{T}_{\alpha}}$ over $A$ such that for all $\nu \in \mathcal{T}_{\alpha}$ with $\operatorname{dom}(\nu)=\alpha, c c_{\nu} \equiv_{A} a b$ (notice that, since $\downarrow^{K}$-dependence is a property witnessed by formulas, one can express these conditions by a type, and using the elements $c_{\emptyset}^{\beta}, \beta<\alpha$, one gets that it is consistent).

Finally, for the limit step, we obtain $\left(c_{\eta}^{\beta}\right)_{\eta \in \mathcal{T}_{\delta}}$ from $\left(c_{\eta}^{\beta}\right)_{\eta \in \mathcal{T}_{\beta}}, 1 \leq \beta<\delta$ in the natural way for any limit ordinal $\delta$.

Now, using the same combinatorial arguments as in 99, we can conclude:

Lemma 4.7. Let $A$ be any set of parameters. If $a \downarrow_{A}^{K} b$, then there is a weak tree Morley sequence $\left(a_{i}\right)_{i<\omega}$ over $A$ which is Ab-indiscernible with $a_{0}=a$.

Notice that if $\left(a_{\eta}\right)_{\eta \in \mathcal{T}_{\omega}}$ is a weak Morley tree over $A$, then if for $\eta_{i} \in \mathcal{T}_{\omega}$ given by $\operatorname{dom}\left(\eta_{i}\right)=[i, \omega)$ and

$$
\eta_{i}(j)=\left\{\begin{array}{lc}
1 & \text { if } i=j \\
0 & \text { otherwise }
\end{array}\right.
$$

$\left(a_{\eta_{i}}\right)_{i<\omega}$ is a Morley sequence over $A$. Hence, repeating the proof of Proposition 5.13 from [9], we get:

Corollary 4.8. (Kim's lemma for weak tree Morley sequences) Let $A$ be any set of parameters. The following are equivalent:

(1) $\varphi(x ; a)$ Kim-divides over $A$.

(2) For some weak tree Morley sequence $\left(a_{i}\right)_{i<\omega}$ over $A$ with $a_{0}=a,\left\{\varphi\left(x ; a_{i}\right)\right.$ : $i<\omega\}$ is inconsistent.

(3) For every weak tree Morley sequence $\left(a_{i}\right)_{i<\omega}$ over $A$ with $a_{0}=a,\left\{\varphi\left(x ; a_{i}\right)\right.$ : $i<\omega\}$ is inconsistent.

Corollary 4.9. $\downarrow^{K}$ satisfies symmetry over arbitrary sets.

Proof. Assume towards a contradiction that $a \downarrow_{A}^{K} b$ and $b \mathcal{L}_{A}^{K} a$. By Lemma 4.7 there is a weak tree Morley sequence $\left\langle a_{i} \mid i<\omega\right\rangle$ over $A$ with $a_{0}=a$ which is $A b$ indiscernible. Since $b \ell_{A}^{K} a$, there is some $\varphi(x ; a) \in \operatorname{tp}(b / A a)$ which Kim-divides 
over A. By Corollary 4.8, $\left\{\varphi\left(x ; a_{i}\right): i<\omega\right\}$ is inconsistent. But $\models \varphi\left(b ; a_{i}\right)$ for all $i<\omega$ by indiscernibility, a contradiction.

\section{3-AMALGAMATiON FOR LASCAR STRONG TYPES}

In this section we will show that in any $\mathrm{NSOP}_{1}$ theory satisfying the existence axiom, Lascar strong types have 3-amalgamation. Throughout this section we assume that $T$ is $\mathrm{NSOP}_{1}$ with existence.

Recall that $a \equiv_{A}^{L} b$ (equivalently $\left.\operatorname{Lstp}(a / A)=\operatorname{Lstp}(b / A)\right)$ if Lascar distance $d_{A}^{L}(a, b)$ is finite, i.e. if there is a finite sequence $a=a_{0}, \ldots, a_{n}=b$ such that $a_{i} a_{i+1}$ begins an $A$-indiscernible sequence for each $i<n$ (iff there is a finite sequence $a=a_{0}, \ldots, a_{n}=b$ and models $M_{i} \supseteq A$ such that $a_{i} \equiv_{M_{i}} a_{i+1}$ for $\left.i<n\right)$. We say $a, b$ have the same KP-type over $A\left(a \equiv_{A}^{K P} b\right)$ if $E(a, b)$ holds for any $A$-typedefinable bounded equivalence relation $E(x, y)$. It follows that $a \equiv_{A}^{L} b$ implies $a \equiv_{A}^{K P} b$. We say $T$ is $G$-compact if KP-types are Lascar types, i.e. the converse holds for any $A$ and any $a, b$ of arbitrary arity. For more on Lascar types see for example [11.

Remark 5.1. $a \equiv_{A}^{L} b$ iff $M d_{A}(a, b)$ is finite, where $M d_{A}(a, b)$ is defined in the same way as Lascar distance of $a, b$ over $A$, except $A$-indiscernible sequences involved in the distance definition are all $A$-Morley sequences: It suffices to observe that for an $A$-indiscernible $\left(a_{0}, a_{1} \in\right) I$, there is $A$-Morley $J$ such that $a_{0} J \equiv_{A} a_{1} J$. But this obviously follows from Fact 2.5(1).

Remark 5.2. By our assumptions and the results from previous sections, we have symmetry: $a \downarrow_{A}^{K} b$ iff $b \downarrow_{A}^{K} a$; Kim-dividing=Kim-forking; and extension: for any partial type $p(x)$ over $B$ not Kim-dividing over a set $A$, there is a completion $q(x) \in S(B)$ of $p$ not Kim-dividing over $A$. In addition, by Proposition 4.5 (or by the same proof as for $\downarrow^{K}$ over a model in [9]), it follows that: if $a \downarrow_{A}^{d} b c$ and $b \downarrow_{A}^{K} c$, then $a b \downarrow_{A}^{K} c$.

Moreover, in the same manner as in [9, Lemma 5.9] we get that if $\left\langle d_{i} \mid i<\omega\right\rangle$ is weak tree Morley over $\emptyset$ with $d_{i}=a_{i} b_{i}$, then so are $\left\langle a_{i} \mid i<\omega\right\rangle$ and $\left\langle d_{n \cdot i} \ldots d_{n \cdot i+n-1}\right|$ $i<\omega\rangle$ for each $n>0$.

In the rest for convenience we take $\emptyset$ as the base set by naming the set; when we say a type Kim-divides, we mean that it does so over $\emptyset$. Now, by a proof similar to that of the weak independence theorem in [9, we obtain the following.

Lemma 5.3. Let $a \downarrow^{K} b$ and $a \downarrow^{K} c$. Then there is e such that ac $\equiv a e, b \downarrow e$, and $a \downarrow^{K}$ be.

Proof. We begin by establishing the following:

Claim. There is $c^{\prime}$ such that $a c^{\prime} \equiv a c$ and $a \downarrow^{K} b c^{\prime}$.

Proof of claim. Due to symmetry, we have $c \downarrow^{K} a$ and $b \downarrow^{K} a$. Hence there is a Morley sequence $J=\left\langle a_{i} \mid i<\kappa\right\rangle$ with $a_{0}=a$ which is $b$-indiscernible, and there is $c^{\prime \prime} \equiv_{a} c$ such that $J$ is $c^{\prime \prime}$-indiscernible. We can assume $\kappa$ is sufficiently large, so by Fact 2.4, there is $J^{\prime} \equiv_{b} J_{\omega}=\left\langle a_{i} \mid i<\omega\right\rangle$ such that $J^{\prime}$ is $b c^{\prime \prime}$-indiscernible. Now if $f$ is some $b$-automorphism sending $J^{\prime}$ to $J_{\omega}$ then $J_{\omega}$ is $a c^{\prime}$-indiscernible where $c^{\prime}=f\left(c^{\prime \prime}\right)$. Hence $b c^{\prime} \downarrow^{K} a$, so $a \downarrow^{K} b c^{\prime}$. The claim is proved. 
Choose $c^{\prime}$ as in the claim and take a Morley sequence $I=\left\langle b_{i} c_{i} \mid i \in \mathbb{Z}\right\rangle$ with $b_{0} c_{0}=b c^{\prime}$. By Proposition 4.5. we can assume $I$ is $a$-indiscernible and $a \downarrow^{K} I$. Let $e=c_{-1}$. Then $a \downarrow^{K} b e$, and since $I$ is a Morley sequence, we have $b \downarrow e$.

Now, we strengthen the lemma.

Lemma 5.4. Let $a \downarrow^{K} b$ and $a \downarrow^{K} c$. Then there is e such that $a c \equiv^{L}$ ae and $b \downarrow e$ and $a \downarrow^{K}$ be.

Proof. By extension and existence for $\downarrow$, there is a model $M$ such that $M \downarrow a b c$. Then by Remark 5.2. we have $M a \downarrow^{K} b$ and $M a \downarrow^{K} c$. Now we apply Lemma 5.3 to $M a, b, c$ to obtain $e$ such that $e \equiv_{M a} c, b \downarrow e$, and $a \downarrow^{K} b e$. Then $a e \equiv^{L} a c$ follows.

Lemma 5.5. (Zig-zag lemma) Let $b \downarrow^{K} c_{0} c_{1}$ and suppose there is a Morley sequence $I=\left\langle c_{i}: i\langle\omega\rangle\right.$. Then there is a weak tree Morley sequence $\left\langle b_{i} c_{i} \mid i<\omega\right\rangle$ such that for all $i, b_{i} c_{i} \equiv b c_{0}$, and for all $j>i, b_{i} c_{j} \equiv b c_{1}$.

Proof. We claim the following first.

Claim. There is Morley $J=\left\langle d_{i} \mid i<\omega\right\rangle \equiv I$ with $d_{0}=c_{0}$ such that $b \downarrow^{K} J$, $b d_{1} \equiv b c_{1}$, and $d_{>0}$ is $b c_{0}$-indiscernible (so $b d_{i} \equiv b c_{1}$ for $i>0$ ).

Proof of claim. Since $I_{2}=\left\langle c_{2 i, 2 i+1}: i\langle\omega\rangle\right.$ is Morley as well, by Proposition 4.5 we can assume that $I_{2}$ is $b$-indiscernible, and $b \downarrow^{K} I_{2}$. Now we can assume the length of $I_{2}$ is a sufficiently large $\kappa$, and consider $J^{\prime}=\left\langle c_{2 i+1} \mid i<\kappa\right\rangle$. Then by Fact 2.4, there is $b c_{0}$-indiscernible $J_{1}=\left\langle e_{i} \mid i<\omega\right\rangle$ such that for each $n<\omega$, $e_{\leq n} \equiv_{b c_{0}} c_{2 i_{0}+1} \ldots c_{2 i_{n}+1}$ for some $i_{0}<\cdots<i_{n}<\kappa$. Now put $J:=c_{0} J_{1}$. Then clearly the claim is satisfied with this $J$.

Now since $d_{>0}$ is Morley and $e_{0} d_{0}$-indiscernible with $b=e_{0}$, we see that $e_{0} d_{0} \downarrow^{K} d_{>0}$, and by extension of $\downarrow^{K}$ there is $e_{1}$ such that $e_{1} d_{\geq 1} \equiv e_{0} J$, and $e_{0} d_{0} \downarrow^{K} e_{1} d_{\geq 1}$. Hence there is Morley $L_{0}=\left\langle e_{0}^{i} d_{0}^{i} \mid i<\omega\right\rangle$ with $e_{0}^{0} d_{0}^{0}=e_{0} d_{0}$ such that $L_{0}$ is $e_{1} d_{\geq 1^{-}}$ indiscernible. Moreover again by Ramsey or Fact 2.4 there is $L_{0} e_{1} d_{1}$-indiscernible sequence $J_{2}$ such that $d_{0} d_{1} J_{2} \equiv J$, so that $L_{0} e_{1} d_{1} \downarrow^{K} J_{2}$. Then by extension there is $e_{2}$ such that $e_{2} J_{2} \equiv e_{1} d_{\geq 1}\left(\equiv e_{0} J\right)$ and $L_{0} e_{1} d_{1} \downarrow^{K} e_{2} J_{2}$. Hence there is Morley $L_{1}=\left\langle L_{0}^{i} e_{1}^{i} d_{1}^{i} \mid i<\omega\right\rangle$ with $L_{0}^{0} e_{1}^{0} d_{1}^{0}=L_{0} e_{1} d_{1}$ such that $L_{1}$ is $e_{2} J_{2^{-}}$ indiscernible. Now let $d_{2}^{\prime}$ be the first component of $J_{2}$. Then again by Fact 2.4 and extension, there are $e_{3}$ and $L_{1} e_{2} d_{2}^{\prime}$-indiscernible $J_{3}$ such that $d_{0} d_{1} d_{2}^{\prime} J_{3} \equiv J$ and $L_{1} e_{2} d_{2}^{\prime} \bigsqcup^{K} e_{3} J_{3}\left(\equiv e_{0} J\right)$. Then there is Morley $L_{2}=\left\langle L_{1}^{i} e_{2}^{i} d_{2}^{i} \mid i<\omega\right\rangle$ with $L_{1}^{0} e_{2}^{0} d_{2}^{0}=L_{1} e_{2} d_{2}$, which is $e_{3} J_{3}$-indiscernible.

We sketch the rest of the proof. Notice that $L_{0} e_{1} d_{1}, L_{1} e_{2} d_{2}^{\prime}, L_{2} e_{3} d_{3}^{\prime}$ are naturally indexed by $\mathcal{T}_{1}, \mathcal{T}_{2}, \mathcal{T}_{3}$, respectively. We iterate this argument for arbitrary large $\alpha$ to get a tree $L_{\alpha} e_{\alpha+1} d_{\alpha+1}^{\prime}$ indexed by $\mathcal{T}_{\alpha+1}$. Notice that each such tree is weakly spread out (see Definition 4.4) by the way of construction. In the process we have kept the following conditions: Let $\left\langle(u v)_{\eta_{\beta}} \mid \beta \leq \alpha+1\right\rangle$ with $\operatorname{dom}\left(\eta_{\beta}\right)=[\beta, \alpha+1)$ be an arbitrary path in the tree. Then $\left\langle v_{\eta_{\beta}} \mid \beta \leq \alpha+1\right\rangle$ has the same EM-type as $J \equiv I$. In particular for any increasing sequence $f(i) \leq \alpha+1$ with $i \in \omega$, we have $\left\langle v_{\eta_{f(i)}} \mid i<\omega\right\rangle \equiv I$. Moreover, for any $\beta$, we have $(u v)_{\eta_{\beta}} \equiv b c_{0}$, and for any $\gamma$ with $\beta<\gamma \leq \alpha+1$, we have $u_{\eta_{\beta}} v_{\eta_{\gamma}} \equiv b c_{1}$. 
Consequently, when we shrink the tree into a weakly Morley tree (as in [9, Section 5]) we can preserve the above conditions and the resulting weakly Morley tree also meets the conditions. Therefore we can find a weak tree Morley sequence described in this lemma.

Theorem 5.6. Let $b \downarrow^{K} c, a \equiv^{L} a^{\prime}$, and $a \downarrow^{K} b$ with $p(x, b)=\operatorname{tp}(a / b), a^{\prime} \downarrow^{K} c$ with $q(x, c)=\operatorname{tp}\left(a^{\prime} / c\right)$. Then $p(x, b) \cup q(x, c)$ does not Kim-divide.

Proof. Since $a \equiv^{L} a^{\prime}$, there is an automorphism $f$ fixing all the Lascar types over $\emptyset$ sending $a$ to $a^{\prime}$. Then clearly, $a \models p(x, b) \cup q\left(x, c^{\prime \prime}\right)$ where $c^{\prime \prime}=f(c)$, so $c \equiv^{L} c^{\prime \prime}$. In particular, $a \downarrow^{K} c^{\prime \prime}$. Due to Lemma 5.4, we can assume $b \downarrow c^{\prime \prime}$ and $a \downarrow^{K} b c^{\prime \prime}$, so $p(x, b) \cup q\left(x, c^{\prime \prime}\right)$ does not Kim-divide. Now applying 5.4 again to $b, c, c^{\prime \prime}$ we can find $c^{\prime}$ such that $b c^{\prime \prime} \equiv b c^{\prime}$ and $c^{\prime \prime} \equiv^{L} c^{\prime}\left(\equiv^{L} c\right)$ such that $b \downarrow^{K} c c^{\prime}$ and $c \downarrow c^{\prime}$. Now as pointed out in Remark 5.1, there are $c^{\prime}=c_{0}, c_{1}, \ldots, c_{n}=c$ such that each pair $c_{i} c_{i+1}$ starts a Morley sequence in $\operatorname{tp}\left(c_{i}\right)$. Moreover, due to extension of $\downarrow^{K}$ applied to $b \downarrow^{K} c c^{\prime}$, we can assume $b \downarrow^{K} c_{\leq n}$. Recall that $p(x, b) \cup q\left(x, c_{0}\right)$ does not Kimdivide $\left(^{*}\right)$, and we shall show that $p(x, b) \cup q\left(x, c_{1}\right)$ does not Kim-divide. (Then the same iterative argument shows that each of $p(x, b) \cup q\left(x, c_{2}\right), \ldots, p(x, b) \cup q\left(x, c_{n}\right)$ does not Kim-divide either, as wanted.)

Now due to Lemma 5.5, there is a weak tree Morley sequence $I=\left\langle b_{i}^{\prime} c_{i}^{\prime}\right| i\langle\omega\rangle$ such that for any $i, b_{i}^{\prime} c_{i}^{\prime} \equiv b c_{0}$, and for $j>i, b_{i}^{\prime} c_{j}^{\prime} \equiv b c_{1}$. Then due to $\left(^{*}\right)$ and Corollary 4.8 .

$$
\bigcup_{i<\omega} p\left(x, b_{i}^{\prime}\right) \cup q\left(x, c_{i}^{\prime}\right)
$$

is consistent. In particular, $\bigcup_{i<\omega} p\left(x, b_{2 i}^{\prime}\right) \cup q\left(x, c_{2 i+1}^{\prime}\right)$ is consistent. Since $\left\langle b_{2 i}^{\prime} c_{2 i+1}^{\prime}\right| i<$ $\omega\rangle$ is weak tree Morley as well (by Remark 5.2 ), we have proved that $p(x, b) \cup q\left(x, c_{1}\right)$ does not Kim-divide.

Lemma 5.7. Assume $b \downarrow^{K} c$. Then for any a there is $e \equiv_{b}^{L}$ a such that $e b \downarrow^{K} c$. Moreover, for any $d$ there is $b^{\prime} \equiv_{c}^{L} b$ such that $b^{\prime} \downarrow^{K} c d$.

Proof. By extension of $\downarrow^{K}$, there is $M$ containing $b$ such that $M \downarrow^{K} c$. Then by extension again there is $e \equiv_{M} a$ (so $e \equiv_{b}^{L} a$ ), such that $e M \downarrow^{K} c$, so $e b \downarrow^{K} c$. Similarly, there is a model $c \in N$ such that $b \downarrow^{K} N$. Then by extension there is $b^{\prime} \equiv_{N} b\left(\right.$ so $\left.b^{\prime} \equiv_{c}^{L} b\right)$, such that $b^{\prime} \downarrow^{K} N d$, hence $b^{\prime} \downarrow^{K} c d$.

Theorem 5.8. (3-amalgamation of Lstp for Kim-dividing) Let $b \downarrow^{K} c, a \equiv^{L} a^{\prime}$, and $a \bigsqcup^{K} b, a^{\prime} \downarrow^{K} c$. Then there is $a^{\prime \prime}$ such that $a^{\prime \prime} \downarrow^{K} b c$ and $a^{\prime \prime} \equiv_{b}^{L} a, a^{\prime \prime} \equiv_{c}^{L} a^{\prime}$. Proof. Due to Lemma 5.7, there is $e \equiv_{b}^{L} a$ such that $e b \downarrow^{K} c$, and there is $e^{\prime} \equiv_{c}^{L} a^{\prime}$ such that $e b \downarrow^{K} c e^{\prime}$. Again by Lemma [5.7, there are $a_{0} \equiv_{b}^{L} a$ and $a_{0}^{\prime} \equiv_{c}^{L} a^{\prime}$ such that $a_{0} \downarrow^{K} e b$ and $a_{0}^{\prime} \downarrow^{K} c e^{\prime}$. Note that $a_{0}^{\prime} \equiv^{L} a^{\prime} \equiv^{L} a \equiv^{L} a_{0}$. Hence by Theorem 5.6. there is $a^{\prime \prime} \models \operatorname{tp}\left(a_{0} / e b\right) \cup \operatorname{tp}\left(a_{0}^{\prime} / c e^{\prime}\right)$ such that $a^{\prime \prime} \downarrow^{K} e b c e^{\prime}$. Moreover since $a^{\prime \prime} \equiv_{e b} a_{0}$ and $e \equiv_{b}^{L} a \equiv_{b}^{L} a_{0}$, it follows that $e \equiv_{b}^{L} a_{0}$ and $a^{\prime \prime} \equiv_{b}^{L} e \equiv_{b}^{L} a$. Similarly we have $a^{\prime \prime} \equiv_{c}^{L} a^{\prime}$ as wanted.

Now by the same argument using 3-amalgamation as in simple theories (see [11]), for any $a \equiv^{L} b$ with $a \downarrow^{K} b$ there is an indiscernible sequence starting with $a, b$. Hence we conclude the following. 
Corollary 5.9. Assume $T$ is $N S O P_{1}$ with existence. Then $T$ is G-compact.

\section{THE SKEW TREE PROPERTY}

Below, we introduce a combinatorial property STP (skew tree property), whose absence turns out to be equivalent to $\mathrm{NSOP}_{1}$ under the assumption of existence.

Definition 6.1. The formula $\varphi(x ; y)$ has the skew tree property if there are indiscernible sequences $\left\langle a_{i}: i<\omega+\omega\right\rangle,\left\langle b_{i}: i<\omega\right\rangle$ satisfying the following properties:

- $\left\{\varphi\left(x ; a_{i}\right): i<\omega+\omega\right\}$ is consistent

- $\left\{\varphi\left(x ; b_{i}\right): i<\omega\right\}$ is inconsistent

- $\left\langle b_{i}: i<\omega\right\rangle$ is $a_{<\omega}$-indiscernible

- $\left\langle a_{\omega+i}: i<\omega\right\rangle$ is $a_{<\omega} b_{>0}$-indiscernible

- $b_{0}=a_{\omega}$

The complete theory $T$ has the skew tree property if some formula does modulo $T$.

Lemma 6.2. (1) If $\varphi$ has $S O P_{1}$ then $\varphi$ has the skew tree property

(2) If $\varphi$ has the skew tree property then $\varphi$ has the tree property.

Proof. (1) Suppose $\varphi$ has $\mathrm{SOP}_{1}$. Then, by Fact 2.9)(3) there is an array $\left(c_{i, 0}, c_{i, 1}\right)_{i \in \omega+\omega+\omega}$ so that

- $\left\{\varphi\left(x ; c_{i, 0}\right): i<\omega+\omega+\omega\right\}$ is consistent

- $\left\{\varphi\left(x ; c_{i, 1}\right): i<\omega+\omega+\omega\right\}$ is inconsistent

- The sequence $\left(\bar{c}_{i}\right)_{i<\omega+\omega+\omega}$ is indiscernible and, for all $i \in \omega+\omega+\omega, c_{i, 0} \equiv_{\bar{c}_{<i}}$ $c_{i, 1}$.

As $c_{\omega+\omega, 0} \equiv_{\bar{c}_{<\omega+\omega}} c_{\omega+\omega, 1}$, there is $\sigma \in \operatorname{Aut}\left(\mathbb{M} / \bar{c}_{<\omega+\omega}\right)$ so that $\sigma\left(c_{\omega+\omega, 0}\right)=c_{\omega+\omega, 1}$. Let $\left\langle a_{i}: i<\omega+\omega\right\rangle$ be defined by $a_{i}=c_{i, 0}$ and $a_{\omega+i}=\sigma\left(c_{\omega+\omega+i, 0}\right)$ for $i<\omega$. Let $\left\langle b_{i}: i<\omega+1\right\rangle$ be defined by $b_{i}=c_{\omega+i, 1}$ for all $i<\omega+1$. In particular $b_{\omega}=c_{\omega+\omega, 1}$. We have $\left\langle a_{i}: i<\omega+\omega\right\rangle$ is an indiscernible sequence, since $\left\langle a_{i}: i<\right.$ $\omega+\omega\rangle=\sigma\left(\left\langle c_{i, 0}: i<\omega\right\rangle \frown\left\langle c_{\omega+\omega+i, 0}: i<\omega\right\rangle\right)$. Similarly, as $\left\langle c_{\omega+\omega+i, 0}: i<\omega\right\rangle$ is $\left\{c_{i, 0}, c_{\omega+i, 1}: i<\omega\right\}$-indiscernible and $\sigma \in \operatorname{Aut}\left(\mathbb{M} / \bar{c}_{<\omega+\omega}\right),\left\langle a_{\omega+i}: i<\omega\right\rangle$ is $a_{<\omega} b_{<\omega}$-indiscernible. As $\left\langle c_{\omega+i, 1}: i<\omega\right\rangle$ is $\left\{c_{i, 0}: i<\omega\right\}$-indiscernible, $\left\langle b_{i}: i<\right.$ $\omega+1\rangle$ is $a_{<\omega}$-indiscernible. Finally, we know $\left\{\varphi\left(x ; a_{i}\right): i<\omega+\omega\right\}$ is consistent, $\left\{\varphi\left(x ; b_{i}\right): i<\omega+1\right\}$ is inconsistent, and $a_{\omega}=b_{\omega}$. This clearly implies the skew tree property.

(2) Immediate from [10, Lemma 2.3].

Define an independence relation $\downarrow^{*}$ by: $a \downarrow_{A}^{*} b$ if there is a Morley sequence $\left\langle b_{i}: i<\omega\right\rangle$ over $A$ with $b_{0}=b$ which is $A a$-indiscernible.

Proposition 6.3. Suppose that with respect to $T, \downarrow^{*}$ satisfies the independence theorem for Lascar strong types over arbitrary sets, that is, given any set $A, c_{0} \equiv_{A}^{L}$ $c_{1}$, with $c_{0} \downarrow_{A}^{*} a, c_{1} \downarrow_{A}^{*} b$, and $a \downarrow_{A}^{*} b$, then there is $c_{*} \models L \operatorname{stp}\left(c_{0} / A a\right) \cup L \operatorname{stp}\left(c_{1} / A b\right)$ with $c_{*} \downarrow_{A}^{*}$ ab. Then $T$ does not have the skew tree property.

Proof. Suppose not, let $\varphi,\left\langle a_{i}: i<\omega+\omega\right\rangle,\left\langle b_{i}: i<\omega\right\rangle$ witness the skew tree property. Let $A=\left\{a_{i}: i<\omega\right\}$. From the definitions, we have $a_{\geq \omega}$ is an $A b_{>0^{-}}$ indiscernible sequence. As $\left\langle a_{i}: i<\omega+\omega\right\rangle$ is indiscernible, we have $a_{\omega+i} \downarrow_{A}^{u} a_{>\omega+i}$ for all $i$. It follows that $a_{\geq \omega}$ is an $\downarrow^{u}$-Morley sequence enumerated reverse over $A$ (hence a Morley sequence over $A$ ). As $a_{\omega}=b_{0}$ and $a_{\geq \omega}$ is $A b_{>0}$-indiscernible, it follows that $b_{>0} \downarrow_{A}^{*} b_{0}$. As $\left\langle b_{i}: i<\omega\right\rangle$ is an $A$-indiscernible sequence, it follows 
that $b_{>i} \downarrow_{A}^{*} b_{i}$ for all $i$. Let $c \models\left\{\varphi\left(x ; a_{i}\right): i<\omega+\omega\right\}$ be a realization. By Ramsey, compactness, and an automorphism, we may assume $\left\langle a_{i}: i<\omega+\omega\right\rangle$ is $c$-indiscernible, hence in particular $c \downarrow_{A}^{*} b_{0}$, since $b_{0}=a_{\omega}$.

By assumption, $\left\{\varphi\left(x ; b_{i}\right): i \leq N\right\}$ is inconsistent for some $N$. We will prove by induction on $k \leq N$ that there is some $c_{k} \models\left\{\varphi\left(x ; b_{i}\right): N-k \leq i \leq N\right\}$ with $c_{k} \downarrow_{A}^{*}\left(b_{i}\right)_{N-k \leq i \leq N}$ to obtain a contradiction. For $k=0$, choose any $c_{0}$ with $c_{0} b_{N} \equiv_{A} c b_{0}$. Now suppose for $k<N$, we have $c_{k} \models\left\{\varphi\left(x ; b_{i}\right): N-\right.$ $k \leq i \leq N\}$ with $c_{k} \downarrow_{A}^{*}\left(b_{i}\right)_{N-k \leq i \leq N}$. We note that $b_{N-k-1}$ and $b_{N-k}$ start an $A$-indiscernible sequence so they have the same Lascar strong type over $A$. Fix $\sigma \in \operatorname{Autf}(\mathbb{M} / A)$ with $\sigma\left(b_{N-k}\right)=b_{N-k-1}$ and let $c^{\prime}=\sigma\left(c_{k}\right)$. Then $c^{\prime} \equiv_{A}^{L}$ $c_{k}, \mathbb{M} \models \varphi\left(c^{\prime} ; b_{N-k-1}\right)$, and $c^{\prime} \bigsqcup_{A}^{*} b_{N-k-1}$. Recall that $\left(b_{i}\right)_{N-k \leq i \leq N} \bigsqcup_{A}^{*} b_{N-k-1}$ so the independence theorem for Lascar strong types implies that there is $c_{*} \models$ $\operatorname{Lstp}\left(c^{\prime} / A b_{N-k-1}\right) \cup \operatorname{Lstp}\left(c_{k} / A\left(b_{i}\right)_{N-k \leq i \leq N}\right)$ with $c_{*} \downarrow_{A}^{*}\left(b_{i}\right)_{N-k-1 \leq i \leq N}$. Note that $c_{*} \models\left\{\varphi\left(x ; b_{i}\right): N-k-1 \leq i \leq N\right\}$ so we may set $c_{k+1}=c_{*}$. Continuing the induction, we find $c_{N} \models\left\{\varphi\left(x ; b_{i}\right): i \leq N\right\}$, contradicting the fact that this set of formulas is inconsistent. This contradiction completes the proof.

Proposition 6.4. Assume $T$ has existence (over any set). Then the following are equivalent.

(1) $T$ is $\operatorname{NSOP}_{1}$.

(2) Kim's lemma holds for Kim-independence over any set.

(3) The independence theorem for Lascar types for $\downarrow^{*}$ holds over any set.

(4) The independence theorem for Lascar types for $\downarrow^{K}$ holds over any set.

(5) Kim-dividing satisfies symmetry over any set.

(6) $T$ does not have STP.

Proof. By Corollary 3.7 we know that (1) and (2) are equivalent. (1) implies (4) by Theorem [5.8. (1) implies (5) by Corollary 4.9, (4) implies (1) by [9, Theorem 6.5] and (5) implies (1) by Fact 2.11. Thus, (1),(2), (4), and (5) are equivalent. Moreover $(3) \Rightarrow(6) \Rightarrow(1)$ is Proposition 6.3 and Lemma 6.2 Finally, assuming (1) (and hence also (2) and (4)), we have $\mathfrak{L}^{*}=\mathfrak{L}^{K}$ by (2) hence (3) follows from (4). This completes the equivalence.

Corollary 6.5. There is a non-simple theory that does not have the skew tree property.

Proof. All of the examples listed in Fact 2.14 are known to be $\mathrm{NSOP}_{1}$ non-simple theories satisfying the existence axiom, and therefore do not have the skew tree property.

Question 6.6. Does the existence axiom hold in any NSOP 1 theory?

Remark 6.7. We observe that if a formula $\varphi(x)$ (over $\emptyset$, say) implies $\phi\left(x, a_{0}\right) \vee$ $\psi\left(x, b_{0}\right)$ and each of $\phi\left(x, a_{0}\right), \psi\left(x, b_{0}\right)$ 2-divides over $\emptyset$ then $T$ has the strict order property: There are indiscernibles $\left\langle a_{i}\right\rangle,\left\langle b_{i}\right\rangle$ witnessing 2-dividing of $\phi\left(x, a_{0}\right)$ and $\psi\left(x, b_{0}\right)$, respectively. Then for $i>0, \varphi(x) \wedge \phi\left(x, a_{i}\right) \models \varphi(x) \wedge \psi\left(x, b_{0}\right)$ and $\varphi(x) \wedge$ $\psi\left(x, b_{i}\right) \models \varphi(x) \wedge \phi\left(x, a_{0}\right)$. Now there is $a_{1}^{\prime}$ such that $a_{1}^{\prime} b_{1} \equiv a_{1} b_{0}$, so $\varphi(x) \wedge \phi\left(x, a_{1}^{\prime}\right)$ implies $\varphi(x) \wedge \phi\left(x, a_{0}\right)$ but not the converse. Since $a_{1}^{\prime} \equiv a_{0}, T$ has the strict order property. Improving this idea to the general case seems difficult. 
Our proof of Kim's lemma relied on Lemma 2.17. This lemma made heavy use of the assumption of non-forking existence for all types over all sets. Consequently, it would be very interesting to know if the following local version could be proved without this assumption:

Question 6.8. Suppose $T$ is $N S O P_{1}, p \in S(A)$, and $\left\langle a_{i}: i<\omega\right\rangle$ and $\left\langle b_{i}: i<\omega\right\rangle$ are both Morley sequences over $A$ in $p$. Is it the case that $\left\{\varphi\left(x ; a_{i}\right): i<\omega\right\}$ is consistent if and only if $\left\{\varphi\left(x ; b_{i}\right): i<\omega\right\}$ is consistent?

Finally, we ask several questions about the skew tree property:

\section{Question 6.9.}

(1) Is $N S O P_{1}$ the same thing as $T$ does not have the skew tree property?

(2) Is the property of not having the skew tree property preserved under reduct?

(3) Is having the skew tree property equivalent to having the skew tree property witnessed by a configuration with $\left\{\varphi\left(x ; b_{i}\right): i<\omega\right\}$ 2-inconsistent?

(4) Does not having the skew tree property imply every complete type has a global non-forking extension?

\section{REFERENCES}

[1] Zoé Chatzidakis. Properties of forking in $\omega$-free pseudo-algebraically closed fields. The Journal of Symbolic Logic, 67(03):957-996, 2002.

[2] Artem Chernikov. Some counterexamples for forking, dividing, invariance. Unpublished note available at https://ffbandf.wordpress.com/tag/invariant-types/.

[3] Artem Chernikov. Theories without the tree property of the second kind. Ann. Pure Appl. Logic, 165(2):695-723, 2014.

[4] Artem Chernikov and Nicholas Ramsey. On model-theoretic tree properties. Journal of Mathematical Logic, page 1650009, 2016.

[5] Gabriel Conant and Alex Kruckman. Independence in generic incidence structures. The Journal of Symbolic Logic, 84(2):750-780, 2019.

[6] Christian d'Elbée. Forking, imaginaries and other features of ACFG. ArXiv preprint arXiv:1812.09378, 2018.

[7] Christian d'Elbée. Generic expansions by a reduct. ArXiv preprint arXiv:1810.11722, 2018.

[8] Mirna Džamonja and Saharon Shelah. On $\triangleleft^{*}$-maximality. Annals of Pure and Applied Logic, 125(1):119-158, 2004.

[9] Itay Kaplan and Nicholas Ramsey. On Kim-independence. J. Eur. Math. Soc. (JEMS), 2019. Accepted, arXiv:1702.03894.

[10] Byunghan Kim. Simplicity, and stability in there. The Journal of Symbolic Logic, 66(02):822836, 2001.

[11] Byunghan Kim. Simplicity theory. Oxford University Press, 2013.

[12] Byunghan Kim, Hyeung-Joon Kim, and Lynn Scow. Tree indiscernibilities, revisited. Arch. Math. Logic, 53(1-2):211-232, 2014.

[13] Byunghan Kim and Anand Pillay. Simple theories. Annals of Pure and Applied Logic, 88(23):149-164, 1997.

[14] Alex Kruckman and Nicholas Ramsey. Generic expansion and skolemization in $\mathrm{NSOP}_{1}$ theories. Annals of Pure and Applied Logic, 169(8):755-774, 2018.

[15] Daniel Lascar and Bruno Poizat. An introduction to forking. Journal of Symbolic Logic, 44(3):330-350, 1979.

[16] Ziv Shami. Definability in low simple theories. Journal of Symbolic Logic, 65(4):1481-1490, 2000 .

[17] Saharon Shelah. Simple unstable theories. Annals of Mathematical Logic, 19(3):177-203, 1980 . 
School of Mathematics, University of Leeds, LS2 9JT, Leeds, UK

E-mail address: J.Dobrowolski@leeds.ac.uk

Department of Mathematics, Yonsei University, 50 Yonsei-ro Seodaemun-gu, Seoul 03722, RePublic of KoreA

E-mail address: bkim@yonsei.ac.kr

Department of Mathematics, UCLA, Los Angeles, USA

E-mail address: nickramsey@math.ucla.edu 